\title{
The Faraday effect revisited: General theory
}

\author{
14th of November, 2005
}

\author{
Horia D. Cornean ${ }^{1}$, Gheorghe Nenciu ${ }^{2}{ }^{3}$, Thomas G. Pedersen ${ }^{4}$
}

\begin{abstract}
This paper is the first in a series revisiting the Faraday effect, or more generally, the theory of electronic quantum transport/optical response in bulk media in the presence of a constant magnetic field. The independent electron approximation is assumed. At zero temperature and zero frequency, if the Fermi energy lies in a spectral gap, we rigorously prove the Widom-Streda formula. For free electrons, the transverse conductivity can be explicitly computed and coincides with the classical result. In the general case, using magnetic perturbation theory, the conductivity tensor is expanded in powers of the strength of the magnetic field $B$. Then the linear term in $B$ of this expansion is written down in terms of the zero magnetic field Green function and the zero field current operator. In the periodic case, the linear term in $B$ of the conductivity tensor is expressed in terms of zero magnetic field Bloch functions and energies. No derivatives with respect to the quasi-momentum appear and thereby all ambiguities are removed, in contrast to earlier work.
\end{abstract}

\section{Introduction}

In sharp contrast with the zero magnetic field case, the analysis of properties of electrons in periodic or random potentials subjected to external magnetic fields is a very challenging problem. The difficulty is rooted in the singular nature of the magnetic interaction: due to a linear increase of the magnetic vector potential, the naive perturbation theory breaks down even at arbitrarily small fields.

To our best knowledge, only the periodic case has been considered in connection with the Faraday effect for bulk systems. The first full scale quantum computation was done by Laura M. Roth [31] (for a review of earlier attempts we direct the reader to this paper). The physical experiment starts by sending a monochromatic light wave, parallel to the $0 z$ direction and linearly polarized in the plane $x 0 z$. When the light enters the material, the polarization plane can change; in fact, there exists a linear relation between the angle $\theta$ of rotation of the plane of polarization per unit length and the transverse component of the conductivity tensor $\sigma_{x y}$ (see formula (1) in 31]). The material is chosen in such

\footnotetext{
${ }^{1}$ Dept. Math., Aalborg University, Fredrik Bajers Vej 7G, 9220 Aalborg, Denmark; e-mail: cornean@math.aau.dk

${ }^{2}$ Dept. Theor. Phys., University of Bucharest, P. O. Box MG11, RO-76900 Bucharest, Romania;

${ }^{3}$ Inst. of Math. "Simion Stoilow" of the Romanian Academy, P. O. Box 1-764, RO-014700 Bucharest, Romania; e-mail: Gheorghe.Nenciu@imar.ro

${ }^{4}$ Dept. Phys. and Nanotech., Pontoppidanstræde 103, 9220 Aalborg, Denmark; e-mail: tgp@physics.aau.dk
} 
a way that when the magnetic field is zero, this transverse component vanishes. When the magnetic field $B$ is turned on, the transverse component is no longer zero. For weak fields one expands the conductivity tensor to first order in $B$ and obtains a formula for the Verdet constant.

Therefore the central object is $\sigma_{x y}(B)$, which depends among other things on temperature, density of the material, and frequency of light. Using a modified Bloch representation, Roth was able to obtain a formula for $\frac{d \sigma_{x y}}{d B}(0)$, and studied how this first order term behaves as a function of frequency, both for metals and semiconductors.

However, the theory in 31] is not free of difficulties. First, it seems almost hopeless to estimate errors or to push the computation to higher orders in $B$. Second, even the first order formula contains terms which are singular at the crossings of the Bloch bands. Accordingly, at the practical level this theory only met a moderate success and alternative formalisms have been used, as for example the celebrated Kohn-Luttinger effective many band Hamiltonian (see [17, 29, 28] and references therein), or tight-binding models [25. Since all these methods have limited applicability, a more flexible approach was still needed.

In the zero magnetic field case, a very successful formalism (see e.g. 27, 16, [2, 9] and references therein) is to use the Green function method. This is based on the fact that the traces involved in computing various physical quantities can be written as integrals involving Green functions. The main aim of our paper is to develop a Green function approach to the Faraday effect, i.e. for the conductivity tensor when a magnetic field is present. Let us point out that the use of Green functions (albeit different from the ones used below) goes back at least to Sondheimer and Wilson 33 in their theory of diamagnetism of Bloch electrons. Aside from the fact that the Green function (i.e. the integral kernel of the resolvent or the semi-group) is easier to compute and control, the main point is that by factorizing out the so called "non integrable phase factor" (or "magnetic holonomy") from the Green function, one can cope with the singularities introduced by the increase at infinity of the magnetic vector potentials. In addition, (as it has already been observed by Schwinger 32 in a QED context) after factorizing out the magnetic holonomy one remains with a gauge invariant quantity which makes the problem of gauge fixing irrelevant. The observation (going back at least to Peierls [26]) that one can use these magnetic phases in order to control the singularity of the magnetic perturbation has been used many times in various contexts (see e.g. 33, 19]). We highlight here the results of Nedoluha 20] where a Green function approach for the magneto-optical phenomena at zero temperature and with the Fermi level in a gap has been investigated.

But the power of this method has only recently been fully exploited in [13. 11], and developed as a general gauge invariant magnetic perturbation theory in 23]. Applied to the case at hand, this theory gives an expansion of the conductivity tensor in terms of the zero magnetic field Green functions. Moreover, it is free of any divergences. A key ingredient in controlling divergences is the exponential decrease of the Green functions with the distance between the arguments, for energies outside the spectrum [10, 22. We stress the fact that since no basis is involved, periodicity is not needed and the theory can also be applied to random systems. Finite systems and/or special geometries (layers) are also allowed. The content of the paper is as follows:

In Section 2 we give a derivation of the conductivity tensor from first prin- 
ciples in the linear response theory. We include it to point out that it coincides with various formulas used before. Although in physics establishing the Kubo formula is considered somehow a triviality, from a mathematical point of view it remains a serious chalenge (see [8]).

Section 3 contains the precise formulation of the thermodynamic limit, stated in Theorem 3.1. We do not give its proof here, but we try to explain why it is true.

Section 4 shows that at the thermodynamic limit, at zero temperature, zero frequency, and for the Fermi energy in a spectral gap, we re-obtain a formula of Streda [34 for the transverse component of the conductivity tensor, known from the Integral Quantum Hall Effect (IQHE). A precise statement and its proof are contained in Theorem 4.1 Moreover, under the proviso that exponentially localized Wannier function exist (see Theorem 4.2), this transverse component vanishes (see also 35, 3] for related results). We stress that this result holds for the whole $\sigma_{x y}(B)$ as long as the magnetic field is not too large, not just for $\frac{d \sigma_{x y}}{d B}(0)$. The vanishing of its first order correction was in fact claimed in formula (50) in 31 .

Section 5 contains the exact quantum computation of $\sigma_{x y}(B)$ for free electrons; in spite of the fact that such a result might be known (and it is known at zero frequency), we were not able to find it in the literature. Interestingly enough, the quantum computation gives the same result as the well known classical computation (when the relaxation time is infinite).

Section [6] contains the core of the paper, which includes the derivation of $\frac{d \sigma_{x y}}{d B}(0)$ for general Bloch electrons. As in the zero magnetic field case, its formula only contains zero magnetic field Green functions and current operators.

Section $[$ deals with periodic systems, and the result of the previous section is written down in terms of zero magnetic field Bloch functions and bands.

At the end we have some conclusions.

The main goal of this paper is to present the strategy, state the results concerning the Verdet constant, and to outline future theoretical and practical problems. Detailed proofs of the thermodynamic limit and of other technical estimates will be given elsewhere.

\section{Preliminaries. The conductivity tensor in the linear response regime}

We begin by fixing the notation used in the description of independent electrons subjected to a constant magnetic field. The units are chosen so that $\hbar=1$. Since we consider spin $1 / 2$ particles, the one particle Hilbert space for a non-confined particle is

$$
\mathcal{H}_{\infty}=L^{2}\left(\mathbb{R}^{3}\right) \oplus L^{2}\left(\mathbb{R}^{3}\right)
$$

with the standard scalar product. Accordingly, all operators below and their integral kernels are $2 \times 2$ matrices in the spin variable. We choose the constant magnetic field of strength $B$ to be oriented along the $z$-axis. Then the one particle Hamiltonian with the spin-orbit coupling included is (see e.g. 31])

$$
H_{\infty}(B)=\frac{1}{2 m} \mathbf{P}(B)^{2}+V+g \mu_{b} B \sigma_{3},
$$


with

$$
\mathbf{P}(B)=-i \nabla-b \mathbf{a}+\frac{1}{2 m c^{2}} \mathbf{s} \wedge(\nabla V)=\mathbf{P}(0)-b \mathbf{a}
$$

where

$$
b=-\frac{e}{c} B
$$

and $\mathbf{a}(\mathbf{x})$ is an arbitrary smooth magnetic vector potential which generates a magnetic field of intensity $B=1$ i.e. $\nabla \wedge \mathbf{a}(\mathbf{x})=(0,0,1)$. The most frequently used magnetic vector potential is the symmetric gauge:

$$
\mathbf{a}_{0}(\mathbf{x})=\frac{1}{2} \mathbf{n}_{3} \wedge \mathbf{x}
$$

where $\mathbf{n}_{3}$ is the unit vector along $z$ axis.

In the periodic case we denote by $\mathcal{L}$ the underlying Bravais lattice, by $\Omega$ its elementary cell and by $\Omega^{*}$ the corresponding Brillouin zone. $|\Omega|$ and $\left|\Omega^{*}\right|$ stand for the volumes of the elementary cell and Brillouin zone respectively. In the absence of the magnetic field one has the well known Bloch representation in terms of Bloch functions:

$$
\Psi_{j}(\mathbf{x}, \mathbf{k})=\frac{1}{\sqrt{\left|\Omega^{*}\right|}} e^{i \mathbf{k} \cdot \mathbf{x}} u_{j}(\mathbf{x}, \mathbf{k}), \quad \mathbf{x} \in \mathbb{R}^{3}
$$

where $u_{j}(\mathbf{x}, \mathbf{k})$ are the normalized to one eigenfunctions of the operator

$$
\begin{gathered}
h(\mathbf{k}) u_{j}(\mathbf{x}, \mathbf{k})=\lambda_{j}(\mathbf{k}) u_{j}(\mathbf{x}, \mathbf{k}) \\
h(\mathbf{k})=\frac{1}{2 m}\left(-i \nabla_{p}+\frac{1}{2 m c^{2}} \mathbf{s} \wedge(\nabla V)+\mathbf{k}\right)^{2}+V, \\
=\frac{1}{2 m}(\mathbf{p}+\mathbf{k})^{2}+V, \quad \mathbf{k} \in \Omega^{*}, \\
\mathbf{p}=-i \nabla_{p}+\frac{1}{2 m c^{2}} \mathbf{s} \wedge(\nabla V),
\end{gathered}
$$

defined in $L^{2}(\Omega) \oplus L^{2}(\Omega)$ with periodic boundary conditions. We label $\lambda_{j}(\mathbf{k})$ in increasing order. We have to remember that, as functions of $\mathbf{k}, \lambda_{j}(\mathbf{k})$ and $u_{j}(\mathbf{x}, \mathbf{k})$ are not differentiable at the crossing points. Since the $\Psi_{j}(\mathbf{x}, \mathbf{k})$ 's form a basis of generalized eigenfunctions, the Green function (i.e. the integral kernel of the resolvent) writes as:

$$
G_{\infty}^{(0)}(\mathbf{x}, \mathbf{y} ; z)=\int_{\Omega^{*}} \sum_{j \geq 1} \frac{\left|\Psi_{j}(\mathbf{x}, \mathbf{k})\right\rangle\left\langle\Psi_{j}(\mathbf{y}, \mathbf{k})\right|}{\lambda_{j}(\mathbf{k})-z} d \mathbf{k},
$$

and it is seen as a matrix in the spin variables. The above formula has to be understood in the formal sense since the series in the right hand side is typically not absolutely convergent, and care is to be taken when interchanging the sum with the integral. Notice however that $G_{\infty}^{(0)}\left(\mathbf{x}, \mathbf{x}^{\prime} ; z\right)$ is a well behaved matrix valued function.

We consider a system of noninteracting electrons in the grand-canonical ensemble. More precisely, we consider a box $\Lambda_{1} \subset \mathbb{R}^{3}$, which contains the origin, and a family of scaled boxes

$$
\Lambda_{L}=\left\{\mathbf{x} \in \mathbb{R}^{3}: \mathbf{x} / L \in \Lambda_{1}\right\} .
$$


The thermodynamic limit will mean $L \rightarrow \infty$, that is when $\Lambda_{L}$ tends to fill out the whole space. The one particle Hilbert space is $\mathcal{H}_{L}:=L^{2}\left(\Lambda_{L}\right) \oplus L^{2}\left(\Lambda_{L}\right)$. The one particle Hamiltonian is denoted by $H_{L}(B)$ and is given by (2.1) with Dirichlet boundary conditions (i.e. the wave-functions in the domain of $H_{L}(B)$ vanish at the surface $\left.\partial \Lambda_{L}\right)$. More precisely, we first define it on $C_{0}^{\infty}\left(\Lambda_{L}\right) \oplus C_{0}^{\infty}\left(\Lambda_{L}\right)$, and then $H_{L}(B)$ will be the Friedrichs extension of this minimal operator. This is indeed possible, because our operator can be written as (up to some irrelevant constants) $-\Delta_{D} I_{2}+W$, where $\Delta_{D}$ is the Dirichlet Laplacian and $W$ is a first order differential operator, relatively bounded to $-\Delta_{D} I_{2}$ (remember that $L<$ $\infty)$ with relative bound zero. The form domain of $H_{L}(B)$ is the Sobolev space $H_{0}^{1}\left(\Lambda_{L}\right) \oplus H_{0}^{1}\left(\Lambda_{L}\right)$, while the operator domain is

$$
\operatorname{Dom}\left(H_{L}(B)\right)=D_{L} \oplus D_{L}, \quad D_{L}:=H^{2}\left(\Lambda_{L}\right) \cap H_{0}^{1}\left(\Lambda_{L}\right) .
$$

Moreover, $H_{L}(B)$ is essentially self-adjoint on $C_{(0)}^{\infty}\left(\overline{\Lambda_{L}}\right)$, i.e. functions with support in $\overline{\Lambda_{L}}$ and indefinitely differentiable in $\Lambda_{L}$ up to the boundary.

We assume that the temperature $T=1 /(k \beta)$ and the chemical potential $\mu$ are fixed by a reservoir of energy and particles. We work in a second quantized setting with an antisymmetric Fock space denoted by $\mathcal{F}_{L}$. Denote the operators in the Fock space with a hat and borrow some notation from the book of Bratelli and Robinson [7]: if $A$ is an operator defined in $\mathcal{H}_{L}$, we denote by $\hat{A}=d \Gamma(A)$ its second quantization in the Fock space. At $t=-\infty$ the system is supposed to be in the grand-canonical equilibrium state of temperature $T$ and chemical potential $\mu$, i.e. the density matrix is

$$
\hat{\rho}_{e}=\frac{1}{\operatorname{Tr}\left(e^{-\beta \hat{K}_{\mu}}\right)} e^{-\beta \hat{K}_{\mu}}
$$

where

$$
\hat{K}_{\mu}=d \Gamma\left(H_{L}(B)-\mu \cdot \mathrm{Id}\right)
$$

is the "grand-canonical Hamiltonian".

The interaction with a classical electromagnetic field is described by a time dependent electric potential

$$
V(\mathbf{x}, t):=\left(e^{i \omega t}+e^{-i \bar{\omega} t}\right) e \mathbf{E} \cdot \mathbf{x}, t \leq 0, \mathbf{x} \in \Lambda_{L} .
$$

so the total time dependent one-particle Hamiltonian is

$$
H(t)=H_{L}(B)+V(t) .
$$

Notice that $e$ near $\mathbf{E}$ is the positive elementary charge. Here we take $\operatorname{Im} \omega<0$ which plays the role of an adiabatic parameter, and insures that there is no interaction in the remote past. Finally, the one-particle current operator is as usual

$$
\mathbf{J}=-e i\left[H_{L}(B), \mathbf{X}\right]=-\frac{e}{m} \mathbf{P}(B),
$$

where $\mathbf{X}$ is the multiplication by $\mathbf{x}$. Note that $\mathbf{J}$ is a well defined operator on the domain of $H_{L}(B)$, because multiplication by any component of $\mathbf{X}$ leaves this domain invariant (see (2.9)). Moreover, since $L<\infty, \mathbf{X}$ is a bounded operator. In fact, $\mathbf{X}$ is the true physical self-adjoint observable, while $\mathbf{P}(B)$ (or $\mathbf{J}$ ) appear when one differentiates the map $t \mapsto e^{i t H_{L}(B)} \mathbf{X} e^{-i t H_{L}(B)}$ in the strong sense on the domain of $H_{L}(B)$. 
We assume that the state of our system is now described by a time-dependent density matrix, $\hat{\rho}(t)$, obtained by evolving $\hat{\rho}_{e}$ from $-\infty$ up to the given time, i.e.

$$
i \partial_{t} \hat{\rho}(t)=[\hat{H}(t), \hat{\rho}(t)], \quad \hat{\rho}(-\infty)=\hat{\rho}_{e} .
$$

Going to the interaction picture and using the Dyson expansion up to the first order, one gets

$$
\hat{\rho}(t=0)=\hat{\rho}_{e}-i \int_{-\infty}^{0}\left[d \Gamma\left(\tilde{V}(s), \hat{\rho}_{e}\right] d s+\mathcal{O}\left(\mathbf{E}^{2}\right),\right.
$$

where

$$
\tilde{V}(s):=e^{i s H_{L}(B)} V(s) e^{-i s H_{L}(B)} .
$$

The current density flowing through our system at $t=0$ is given by (see (2.16) ):

$$
\begin{aligned}
\mathbf{j} & =\frac{1}{\left|\Lambda_{L}\right|} \operatorname{Tr}_{\mathcal{F}_{L}}(\hat{\rho}(0) \hat{\mathbf{J}})=\frac{1}{\left|\Lambda_{L}\right|} \operatorname{Tr}_{\mathcal{F}_{L}}\left(\hat{\rho}_{e} \hat{\mathbf{J}}\right) \\
& -\frac{i}{\left|\Lambda_{L}\right|} \operatorname{Tr}_{\mathcal{F}_{L}}\left(\int_{-\infty}^{0}\left[d \Gamma(\tilde{V}(s)), \hat{\rho}_{e}\right] \hat{\mathbf{J}} d s\right)+\mathcal{O}\left(\mathbf{E}^{2}\right) .
\end{aligned}
$$

In evaluating the r.h.s. of (2.18) we use the well known fact that traces over the Fock space can be computed in the one-particle space (see Proposition 5.2.23 in 7]):

$$
\operatorname{Tr}_{\mathcal{F}_{L}}\left\{\hat{\rho}_{e} d \Gamma(A)\right\}=\operatorname{Tr}_{\mathcal{H}_{L}}\left\{f_{F D}\left(H_{L}(B)\right) A\right\}
$$

where $f_{F D}$ is the Fermi-Dirac one-particle distribution function:

$$
f_{F D}(x):=\frac{1}{e^{\beta(x-\mu)}+1}, \quad x \in \mathbb{R}, \beta>0, \mu \in \mathbb{R}
$$

Plugging (2.19) into (2.18), the identity $[d \Gamma(A), d \Gamma(B)]=d \Gamma([A, B])$, the invariance of trace under cyclic permutations and ignoring the quadratic correction in $\mathbf{E}$ one arrives at

$$
\begin{aligned}
\mathbf{j} & =\frac{1}{\left|\Lambda_{L}\right|} \operatorname{Tr}_{\mathcal{H}_{L}}\left\{f_{F D}\left(H_{L}(B)\right) \mathbf{J}\right\} \\
& -\frac{i}{\left|\Lambda_{L}\right|} \frac{e}{m} \operatorname{Tr}_{\mathcal{H}_{L}}\left(\int_{-\infty}^{0}[\tilde{V}(s), \mathbf{P}(B)] f_{F D}\left(H_{L}(B)\right) d s\right) .
\end{aligned}
$$

The first term in (2.21) is always zero because of the identity (trace cyclicity again)

$$
\operatorname{Tr}_{\mathcal{H}_{L}}\left\{\left[H_{L}(B), \mathbf{X}\right] f_{F D}\left(H_{L}(B)\right)\right\}=\operatorname{Tr}_{\mathcal{H}_{L}}\left\{\left[f_{F D}\left(H_{L}(B)\right), H_{L}(B)\right] \mathbf{X}\right\}=0 .
$$

which is nothing but the fact that the current vanishes on an equilibrium state. Note that these operations under the trace sign are quite delicate, since unbounded operators are involved. Let us for once give a complete proof to (2.22). We have the identity between bounded operators (consider the first component $\left.X_{1}\right)$ :

$$
\left[H_{L}(B), X_{1}\right] f_{F D}\left(H_{L}(B)\right)=H_{L}(B) X_{1} f_{F D}\left(H_{L}(B)\right)-X_{1} H_{L}(B) f_{F D}\left(H_{L}(B)\right) \text {. }
$$


Remember that $X_{1}$ is a bounded operator in the box, and preserves the domain of $H_{L}(B)$. This means that the operator $O_{L}=\left(H_{L}(B)+i\right) X_{1}\left(H_{L}(B)+i\right)^{-1}$ is bounded. Hence we can write

$$
H_{L}(B) X_{1} f_{F D}\left(H_{L}(B)\right)=\left[1-i\left(H_{L}(B)+i\right)^{-1}\right] O_{L}\left[H_{L}(B)+i\right] f_{F D}\left(H_{L}(B)\right) .
$$

Now the operator $\left[H_{L}(B)+i\right] f_{F D}\left(H_{L}(B)\right)$ still is trace class due to the exponential decay of $f_{F D}$, while $\left[1-i\left(H_{L}(B)+i\right)^{-1}\right]$ and $O_{L}$ are bounded. Thus $H_{L}(B) X_{1} f_{F D}\left(H_{L}(B)\right)$ is trace class and we can compute its trace using the complete eigenbasis of $H_{L}(B)$, which gives the same result as for the other operator $X_{1} H_{L}(B) f_{F D}\left(H_{L}(B)\right)$. Thus (2.22) is proved.

Using (2.12) and (2.17) one can write

$$
j_{\alpha}=\sum_{\beta=1}^{3}\left\{\sigma_{\alpha \beta}(\omega)+\sigma_{\alpha \beta}(-\bar{\omega})\right\} E_{\beta}, \quad \alpha \in\{1,2,3\}, \Im(\omega)<0,
$$

where the conductivity tensor is given by

$$
\begin{aligned}
& \sigma_{\alpha \beta}(B, \omega)= \\
& -\frac{i}{\left|\Lambda_{L}\right|} \frac{e^{2}}{m} \operatorname{Tr}_{\mathcal{H}_{L}} \int_{-\infty}^{0}\left[e^{i s H_{L}(B)} x_{\beta} e^{-i s H_{L}(B)}, P_{\alpha}(B)\right] f_{F D}\left(H_{L}(B)\right) e^{i s \omega} d s .
\end{aligned}
$$

Performing an integration by parts, using the formulas $i\left[H_{L}(B), x_{\beta}\right]=P_{\beta}(B) / m$ and $i\left[P_{\alpha}(B), x_{\beta}\right]=\delta_{\alpha \beta}$ one arrives at

$$
\begin{gathered}
\sigma_{\alpha \beta}(B, \omega)=\frac{1}{\left|\Lambda_{L}\right|} \frac{e^{2}}{i m \omega}\left\{\delta_{\alpha \beta} \operatorname{Tr}\left(f_{F D}\left(H_{L}(B)\right)\right)\right. \\
\left.+\frac{i}{m} \operatorname{Tr} \int_{-\infty}^{0} e^{i s\left(\omega+H_{L}(B)\right)} P_{\beta}(B) e^{-i s H_{L}(B)}\left[P_{\alpha}(B), f_{F D}\left(H_{L}(B)\right)\right] d s\right\}
\end{gathered}
$$

and this coincides (at least at the formal level) with formula (5) in 31. Notice that from now on, we write just $\operatorname{Tr}$ when we perform the trace, since we only work in the one-particle space.

Since we are interested in the Faraday effect, and we assume that the magnetic field $\mathbf{B}$ is parallel with the $z$ axis, we will only consider the transverse conductivity $\sigma_{12}(B, \omega)$. Hence the first term vanishes. We now perform the integral over $s$ with the help of Stone's formula followed by a deformation of the contour (paying attention not to hit the singularities of $f_{F D}(z)$ or to make the integral over $s$ divergent

$$
f_{F D}\left(H_{L}(B)\right) e^{i s\left(H_{L}(B)+\eta\right)}=\frac{i}{2 \pi} \int_{\Gamma_{\omega}} f_{F D}(z) e^{i s(z+\eta)}\left(H_{L}(B)-z\right)^{-1} d z .
$$

where $\eta$ is either 0 or $\omega$, the contour is counter-clockwise oriented and given by

$$
\Gamma_{\omega}=\{x \pm i d: a \leq x<\infty\} \bigcup\{a+i y:-d \leq y \leq d\}
$$

with

$$
d=\min \left\{\frac{\pi}{2 \beta}, \frac{|\operatorname{Im} \omega|}{2}\right\}
$$


and $a+1$ lies below the spectrum of $H_{L}(B)$. As a final result one gets

$$
\begin{aligned}
& \sigma_{12}(B, \omega)=-\frac{e^{2}}{2 \pi m^{2} \omega\left|\Lambda_{L}\right|} \\
& \cdot \operatorname{Tr} \int_{\Gamma_{\omega}} f_{F D}(z)\left\{P_{1}(B)\left(H_{L}(B)-z\right)^{-1} P_{2}(B)\left(H_{L}(B)-z-\omega\right)^{-1}\right. \\
& +z \rightarrow z-\omega\} d z=: \frac{e^{2}}{m^{2} \omega} a_{L}(B, \omega)
\end{aligned}
$$

where " $z \rightarrow z-\omega$ " means a similar term where we exchange $z$ with $z-\omega$. Now one can see that by inserting the eigenbasis of $H_{L}(B)$ one obtains the well known formula derived from semi-classical radiation theory (see e.g. formula (4) in [31]).

\section{Gauge invariance and existence of the ther- modynamic limit}

Up to now the system was confined in a box $\Lambda_{L}$. As is well known (see e.g. 31]) a direct evaluation of (2.30) (or previous formulas equivalent to it including formula (4) in Roth's paper) is out of reach: the eigenvalues and eigenstates of $H(B)$ are rather complicated (even in the thermodynamic limit $\Lambda_{L} \rightarrow \mathbb{R}^{3}$ ) and at the same time the Bloch representation is plagued by singular matrix elements of the magnetic vector potential. Roth used a modified magnetic Bloch representation in [30] and derived a formula for the linear term in $B$ of (2.30) in terms of the zero magnetic field Bloch representation. Still, her procedure is not free of difficulties since it involves $\nabla_{\mathbf{k}} u_{j}(\mathbf{x}, \mathbf{k})$ which might not exist at crossing points. In addition, it seems almost hopeless to control the errors or to push computations to the second order in $B$ which would describe the Cotton-Mouton effect for example.

In what follows, we shall outline another route of evaluating (2.30) which is mathematically correct, systematic, and completely free of the above difficulties. There are two basic ideas involved. The first one (going back at least to Sondheimer and Wilson [33] in their theory of diamagnetism) consists in writing the trace in (2.30) as integrals over $\Lambda_{L}$ of corresponding integral kernels. This is nothing but the well known Green function approach (see e.g. [15]) which has been very successful in computing optical and magneto-optical properties of solids (see e.g. 27], 16], 2]) in the absence of an external magnetic field. The point is that the integral kernels are on one hand easier to control and compute, and on the other hand they do not require periodicity. Moreover, this approach proved to be essential in deriving rigorous results concerning the diamagnetism of free electrons 11, 11 and actually we expect the methods of the present paper to simplify the theory of diamagnetism of Bloch electrons as well.

However, when applying Green function approach in the presence of an external magnetic field one hits again the divergences caused by the linear increase of the magnetic vector potential: naively, at the first sight $a_{L}(B, \omega)$ is not bounded in the thermodynamic limit $L \rightarrow \infty$ but instead grows like the second power of $L$. It was already observed in [1] that these divergent terms vanish identically due to some identities coming from gauge invariance. 
This is indeed the case and the main point of this paper is to show, following the developments in 13, 11, 23, that factorizing the so called "non-integrable phase factor" from the Green function (the integral kernel of $\left.\left(H_{L}(B)-\zeta\right)^{-1}\right)$ allows, at the same time, to eliminate the divergences coming from the increase of the magnetic vector potential and to obtain a controlled expansion in powers of $B$. In addition, this leads to expressions of $a_{L}(B, \omega)$ which are manifestly gauge invariant.

For an arbitrary pair of points $\mathbf{x}, \mathbf{y} \in \Lambda_{L}$ consider the "magnetic phase" associated with the magnetic vector potential $\mathbf{a}(\mathbf{u})$ defined as the path integral on the line linking $\mathbf{y}$ and $\mathbf{x}$ :

$$
\phi_{\mathbf{a}}(\mathbf{x}, \mathbf{y})=\int_{\mathbf{y}}^{\mathbf{x}} \mathbf{a}(\mathbf{u}) \cdot d \mathbf{u} .
$$

The magnetic phase satisfies the following crucial identity: for every fixed $\mathbf{c}$

$$
e^{-i b \phi_{\mathbf{a}}(\mathbf{x}, \mathbf{c})} \mathbf{P}(B) e^{i b \phi_{\mathbf{a}}(\mathbf{x}, \mathbf{c})}=\mathbf{P}(0)-b \mathbf{A}(\mathbf{x}-\mathbf{c}) .
$$

where $\mathbf{A}(\mathbf{x})=\frac{1}{2} \mathbf{n}_{3} \wedge \mathbf{x}$, i.e. irrespective of the choice of $\mathbf{a}(\mathbf{x})$,

$$
\mathbf{A}(\mathbf{x}-\mathbf{c})=\frac{1}{2} \mathbf{n}_{3} \wedge(\mathbf{x}-\mathbf{c})
$$

is the symmetric (transverse, Poincaré) gauge with respect to c.

Write now the Green function (as a $2 \times 2$ matrix in the spin space)

$$
G_{L}(\mathbf{x}, \mathbf{y} ; \zeta)=\left(H_{L}-\zeta\right)^{-1}(\mathbf{x}, \mathbf{y})
$$

in the factorized form

$$
G_{L}(\mathbf{x}, \mathbf{y} ; \zeta)=e^{i b \phi_{\mathbf{a}}(\mathbf{x}, \mathbf{y})} K_{L}(\mathbf{x}, \mathbf{y} ; \zeta)
$$

It is easy to check that while $G_{L}(\mathbf{x}, \mathbf{y} ; \zeta)$ is gauge dependent, $K_{L}(\mathbf{x}, \mathbf{y} ; \zeta)$ is gauge independent i.e. the whole gauge dependence of $G_{L}(\mathbf{x}, \mathbf{y} ; \zeta)$ is contained in the phase factor $e^{i b \phi_{\mathbf{a}}(\mathbf{x}, \mathbf{y})}$. Plugging the factorization (3.5) into the integrand of the r.h.s. of (2.30), using (3.2) and (3.3), one obtains that its integral kernel writes as

$$
\begin{aligned}
& \mathcal{A}_{s, s^{\prime}}^{L}\left(\mathbf{x}, \mathbf{x}^{\prime}\right)=e^{i b \phi_{\mathbf{a}}\left(\mathbf{x}, \mathbf{x}^{\prime}\right)} \\
& \cdot \int_{\Gamma_{\omega}} d z f_{F D}(z) \sum_{\sigma=1}^{2} \int_{\Lambda_{L}} d \mathbf{y} e^{i b \Phi\left(\mathbf{x}, \mathbf{y}, \mathbf{x}^{\prime}\right)}\left\{\left[\left(P_{1, \mathbf{x}}(0)-b A_{1}(\mathbf{x}-\mathbf{y})\right) K_{L}(\mathbf{x}, \mathbf{y} ; z)\right]_{s, \sigma}\right. \\
& \left.\cdot\left[\left(P_{2, \mathbf{y}}(0)-b A_{2}(\mathbf{x}-\mathbf{y})\right) K_{L}\left(\mathbf{y}, \mathbf{x}^{\prime} ; z+\omega\right)\right]_{\sigma, s^{\prime}}+z \rightarrow z-\omega\right\}
\end{aligned}
$$

where

$$
\Phi\left(\mathbf{x}, \mathbf{y}, \mathbf{x}^{\prime}\right)=\phi_{\mathbf{a}}(\mathbf{x}, \mathbf{y})+\phi_{\mathbf{a}}\left(\mathbf{y}, \mathbf{x}^{\prime}\right)+\phi_{\mathbf{a}}\left(\mathbf{x}^{\prime}, \mathbf{x}\right)
$$

is the flux of the magnetic field $(0,0,1)$ through the triangle $\Delta\left(\mathbf{x}, \mathbf{y}, \mathbf{x}^{\prime}\right)$. Now the fact that there are no long range divergences in the formula for $\mathcal{A}_{s, s^{\prime}}\left(\mathbf{x}, \mathbf{x}^{\prime}\right)$ follows from the exponential decay of Green functions [10] (see also 22]): for $\zeta$ outside the spectrum of $H$ there exists $m(\zeta)>0$ such that as $|\mathbf{x}-\mathbf{y}| \rightarrow \infty$

$$
\left|K_{L}(\mathbf{x}, \mathbf{y} ; \zeta)\right|=\left|G_{L}(\mathbf{x}, \mathbf{y} ; \zeta)\right| \sim e^{-m(\zeta)|\mathbf{x}-\mathbf{y}|} .
$$


It can be proved (the technical details which are far from being simple will be given elsewhere) that $\mathcal{A}_{s, s^{\prime}}^{L}\left(\mathbf{x}, \mathbf{x}^{\prime}\right)$ is jointly continuous and moreover outside a thin region near the surface of $\Lambda_{L}$ one can replace it by the integral kernel $\mathcal{A}_{s, s^{\prime}}^{\infty}\left(\mathbf{x}, \mathbf{x}^{\prime}\right)$ of the corresponding operator on the whole $\mathbb{R}^{3}$. Accordingly, up to surface corrections:

$$
a_{L}(B, \omega) \approx-\frac{1}{2 \pi\left|\Lambda_{L}\right|} \sum_{s=1}^{2} \int_{\Lambda_{L}} \mathcal{A}_{s, s}^{\infty}(\mathbf{x}, \mathbf{x}) d \mathbf{x} .
$$

Notice that due to the fact that $\Phi(\mathbf{x}, \mathbf{y}, \mathbf{x})=\phi_{\mathbf{a}}(\mathbf{x}, \mathbf{x})=0$ the phase factors appearing in (3.6) reduce to unity in (3.8).

In the periodic case, from the fact that in the symmetric gauge the Hamiltonian $H_{\infty}(B)$ commutes with the magnetic translations (actually one can define magnetic translations for an arbitrary gauge, just first make the gauge transformation relating $\mathbf{a}(\mathbf{x})$ to $\mathbf{A}(\mathbf{x}))$ generated by $\mathcal{L}$, it follows that for $\vec{\gamma} \in \mathcal{L}$ we have:

$$
K_{\infty}(\mathbf{x}+\vec{\gamma}, \mathbf{y}+\vec{\gamma} ; \zeta)=K_{\infty}(\mathbf{x}, \mathbf{y} ; \zeta)
$$

which implies that

$$
\mathcal{A}_{s, s}^{\infty}(\mathbf{x}+\vec{\gamma}, \mathbf{x}+\vec{\gamma})=\mathcal{A}_{s, s}^{\infty}(\mathbf{x}, \mathbf{x})
$$

is periodic with respect to $\mathcal{L}$, hence up to surface corrections:

$$
a_{L}(B, \omega) \approx a(B, \omega)=-\frac{1}{2 \pi|\Omega|} \sum_{s=1}^{2} \int_{\Omega} \mathcal{A}_{s, s}^{\infty}(\mathbf{x}, \mathbf{x}) d \mathbf{x} .
$$

Therefore, the transverse conductivity writes as

$$
\sigma_{12}(B, \omega)=\frac{e^{2}}{m^{2} \omega} a(B, \omega)
$$

with $a(B, \omega)$ given by the r.h.s. of (3.9).

A precise formulation of this result is contained in the following theorem:

Theorem 3.1. Assume for simplicity that $\Omega$ is the unit cube in $\mathbb{R}^{3}$. The above defined transverse component of the conductivity tensor admits the thermodynamic limit; more precisely:

i. The following operator defined by a $B\left(L^{2} \oplus L^{2}\right)$-norm convergent Riemann integral,

$$
\begin{aligned}
F_{L} & :=-\frac{1}{2 \pi} \int_{\Gamma_{\omega}} f_{F D}(z)\left\{P_{1}(B)\left(H_{L}(B)-z\right)^{-1} P_{2}(B)\left(H_{L}(B)-z-\omega\right)^{-1}\right. \\
& +z \rightarrow z-\omega\} d z
\end{aligned}
$$

is in fact trace-class, and $\sigma_{12}^{(L)}(B, \omega)=\frac{e^{2}}{m^{2} \omega\left|\Lambda_{L}\right|} \operatorname{Tr}\left(F_{L}\right)$.

ii. Consider the operator $F_{\infty}$ defined by the same integral but with $H_{\infty}(B)$ instead of $H_{L}(B)$, and defined on the whole space. Then $F_{\infty}$ is an integral operator, with a kernel $\mathcal{A}_{s, s^{\prime}}^{\infty}\left(\mathbf{x}, \mathbf{x}^{\prime}\right)$ jointly continuous on its spatial variables. Moreover, the function defined by $\mathbb{R}^{3} \ni \mathbf{x} \rightarrow s_{B}(\mathbf{x}):=\sum_{s=1}^{2} \mathcal{A}_{s, s}^{\infty}(\mathbf{x}, \mathbf{x}) \in \mathbb{C}$ is continuous and periodic with respect to $\mathbb{Z}^{3}$.

iii. The thermodynamic limit exists:

$$
\sigma_{12}^{(\infty)}(B, \omega):=\lim _{L \rightarrow \infty} \sigma_{12}^{(L)}(B, \omega)=\frac{e^{2}}{m^{2} \omega|\Omega|} \int_{\Omega} s_{B}(\mathbf{x}) d \mathbf{x} .
$$


The proof of this theorem will be given elsewhere 14.

\section{The zero frequency limit at $T=0$ : a rigorous proof of the Widom-Streda formula for semi- conductors}

Doing some very formal computations one can show that at $T=0$ and $\omega=0$, $\sigma_{12}(B, \omega)$ as given by (3.9) and (3.10) coincide with the formula for the quantized Hall conductivity (see e.g. formulas (5),(6) in 34) which in turn gives (again at the heuristic level) the well known Widom-Streda formula. The original derivation has little mathematical rigor, in particular because it assumes some very strong assumptions on the existence and regularity of $\left(H_{\infty}(B)-\lambda+i 0\right)^{-1}$ as a function of $\lambda \in \mathbb{R}$. These assumptions are clearly not true in many situations.

Here we will show how the Widom-Streda formula can be rigorously obtained when the Fermi energy lies in a spectral gap. The problem in which the Fermi energy is in the spectrum, remains open. Now assume that for some $B$, the chemical potential $\mu$ lies in a spectral gap of $H_{\infty}(B)$. More precisely, throughout this section we suppose that $\left(d_{1}, d_{2}\right) \subset \rho\left(H_{\infty}(B)\right)$ with $d_{1}<d_{2}$, and take $\mu \in\left(d_{1}, d_{2}\right)$. For simplicity, assume that $\mu=\frac{d_{1}+d_{2}}{2}$. This is the typical situation for semiconductors and/or isolators. In the absence of spin, the Widom-Streda formula roughly states that:

$$
\sigma_{12}(B, T=0, \omega=0)=\left.e c \frac{\partial N(B, E)}{\partial B}\right|_{E=\mu},
$$

where $N(B, E)$ is the integrated density of states up to the energy $E$. When the spin is present (this was not considered by Streda), this formula is slightly changed. If we denote by $B_{1}$ the $B$ multiplying the spin matrix $\sigma_{3}$ in our Hamiltonian (2.1), and with $B_{2}$ the $B$ near $\mathbf{A}$, then in fact we have

$$
\sigma_{12}(B, T=0, \omega=0)=\left.e c \frac{\partial N\left(B_{1}, B_{2}, E\right)}{\partial B_{2}}\right|_{E=\mu, B_{1}=B_{2}=B} .
$$

In the rest of this section we give a rigorous (but still not fully technical) proof of (4.2).

Theorem 4.1. Consider the conductivity at the thermodynamic limit given in (3.12), and drop the superscript $\infty$. Then if we first take the limit $T \searrow 0$, and after that $\omega \rightarrow 0$, we get:

$$
\lim _{\omega \rightarrow 0} \lim _{T \rightarrow 0} \sigma_{12}(B, T, \omega)=\left.e c \frac{\partial}{\partial B_{2}} \frac{1}{|\Omega|} \sum_{s=1}^{2} \int_{\Omega} \Pi_{s, s}^{B}(\mathbf{x}, \mathbf{x}) d \mathbf{x}\right|_{B_{1}=B_{2}=B},
$$

where

$$
\Pi^{B}=\frac{i}{2 \pi} \int_{\Gamma} \frac{1}{H_{\infty}(B)-z} d z
$$

with a positively oriented contour $\Gamma$ enclosing the spectrum of $H_{\infty}(B)$ below $\mu$, i.e. $\Pi^{B}$ is the Fermi projection onto the subspace of "occupied" states at $T=0$. 


\section{Remarks:}

1. Streda did not consider spin in his work 34 and in this case the derivative with respect to the magnetic field appears in the r.h.s. of (4.3).

2. While it is not clear that $\Pi^{B}(\mathbf{x}, \mathbf{x})$ is well defined $\left(\left(H_{\infty}(B)-z\right)^{-1}(\mathbf{x}, \mathbf{x})\right.$ does not exist!) this can be seen by writing for some $a \in \rho\left(H_{\infty}(B)\right)$ :

$$
\begin{aligned}
\Pi^{B} & =\frac{1}{2 \pi} \int_{\Gamma}\left(\left(H_{\infty}(B)-z\right)^{-1}-\left(H_{\infty}(B)-a\right)^{-1} d z\right. \\
& =\frac{1}{2 \pi} \int_{\Gamma}(z-a)\left(H_{\infty}(B)-z\right)^{-1}\left(H_{\infty}(B)-a\right)^{-1} d z .
\end{aligned}
$$

Each resolvent has a polar integral kernel with a $1 /\left|\mathbf{x}-\mathbf{x}^{\prime}\right|$ singularity, and the product of two resolvents will have a continuous kernel. In fact we can repeat this trick and obtain products of as many resolvents as we want, thus further improving the regularity of the integral kernel. Technical details will be given elsewhere. Actually this kind of argument can be used to show that all operators defined by integrals over complex contours have jointly continuous integral kernels.

3. Although the order of limits in (4.3) is important for the argument below, it might be possible (at least under additional conditions on the spectrum of $\left.H_{\infty}(B)\right)$ to interchange the order of limits. The important fact is that the thermodynamic limit has to be taken first: great care is to be taken when defining currents in the static limit for finite systems (for a discussion of this point in a related context see [24].

4. The result is valid for arbitrary magnetic field $B$ and establishes the connection between the Hall conductivity and the Faraday effect. However, the quantum Hall effect requires high magnetic fields while the Faraday effect is usually considered at low magnetic fields.

Proof. We start from the conductivity in the thermodynamic limit as given by Theorem 3.1

$$
\sigma_{12}(B, T, \omega)=-\frac{e^{2}}{2 \pi m^{2} \omega|\Omega|} \sum_{s=1}^{2} \int_{\Omega}\left[\int_{\Gamma_{\beta, \omega}} f_{F D}(z) \Sigma(z, \omega) d z\right]_{s, s}(\mathbf{x}, \mathbf{x}) d \mathbf{x},
$$

where

$$
\Sigma(z, \omega):=P_{1}(B)\left(H_{\infty}(B)-z\right)^{-1} P_{2}(B)\left(H_{\infty}(B)-z-\omega\right)^{-1}+(z \rightarrow z-\omega) .
$$

Since we made the assumption that $\left(d_{1}, d_{2}\right) \subset \rho\left(H_{\infty}(B)\right.$, then for $|\omega|<$ $\frac{d_{2}-d_{1}}{4}$ the integral over $z$ on the contour $\Gamma_{\beta, \omega}$ can be replaced with the integral on the contour $\Gamma_{\beta, \omega}^{1} \cup \Gamma_{\beta, \omega}^{2}$ where (see also (2.28) and (2.29)):

$$
\begin{aligned}
\Gamma_{\beta, \omega}^{1}=\left\{x \pm i d: a \leq x \leq d_{1}+\frac{d_{2}-d_{1}}{4}\right\} \bigcup\{a+i y:-d \leq y \leq d\} \\
\bigcup\left\{d_{1}+\frac{d_{2}-d_{1}}{4}+i y:-d \leq y \leq d\right\}
\end{aligned}
$$


and

$$
\begin{aligned}
\Gamma_{\beta, \omega}^{2}= & \left\{x \pm i d: x \geq d_{2}-\frac{d_{2}-d_{1}}{4}\right\} \\
& \bigcup\left\{d_{2}-\frac{d_{2}-d_{1}}{4}+i y:-d \leq y \leq d\right\} .
\end{aligned}
$$

Accordingly, one can rewrite $\sigma_{12}(B, T, \omega)$ as

$$
\begin{aligned}
& \sigma_{12}(B, T, \omega)=-\frac{e^{2}}{2 \pi m^{2} \omega|\Omega|} \sum_{s=1}^{2} \int_{\Omega} \\
& \left\{\int_{\Gamma_{\beta, \omega}^{1}} \Sigma(z, \omega) d z\right. \\
& \left.+\int_{\Gamma_{\beta, \omega}^{1}}\left(f_{F D}(z)-1\right) \Sigma(z, \omega) d z+\int_{\Gamma_{\beta, \omega}^{2}} f_{F D}(z) \Sigma(z, \omega) d z\right\}_{s, s}(\mathbf{x}, \mathbf{x}) d \mathbf{x} .
\end{aligned}
$$

Note that since the singularities of $f_{F D}(z)$ lie on $\frac{c+d}{2}+i y, y \in(-\infty, \infty)$, one can take $\Gamma_{\beta, \omega}^{j}$ independent of $\beta$ i.e. one can take $d=\frac{|\Im(\omega)|}{2}$ in (2.29). At this point we take the limit $\beta \rightarrow \infty$. Since on $\Gamma_{\omega}^{2}$ we have $\left|f_{F D}(z)\right| \leq 2 \exp \left[-\beta\left(x-\frac{d_{1}+d_{2}}{2}\right)\right]$, and on $\Gamma_{\omega}^{1}$ we have that $\left|f_{F D}(z)-1\right| \leq 2 \exp \left[-\beta\left(\frac{d_{1}+d_{2}}{2}-x\right)\right]$, the last two terms in (4.10) vanish in the zero temperature limit (full details about the control of various integral kernels will be given elsewhere). Hence we get:

$$
\sigma_{12}(B, T=0, \omega)=-\frac{e^{2}}{2 \pi m^{2} \omega|\Omega|} \sum_{s=1}^{2} \int_{\Omega}\left\{\int_{\Gamma_{\omega}^{1}} \Sigma(z, \omega) d z\right\}_{s, s}(\mathbf{x}, \mathbf{x}) d \mathbf{x} .
$$

An application of the Cauchy residue theorem shows that the two terms of $\Sigma(z, \omega)$ (see 4.7) will combine in the above integral and give

$$
\begin{aligned}
& \sigma_{12}(B, T=0, \omega)=-\frac{e^{2}}{2 \pi m^{2} \omega} \frac{1}{|\Omega|} \sum_{s=1}^{2} \\
& \int_{\Omega}\left[\int_{\Gamma} P_{1}(B)\left(H_{\infty}(B)-z+\omega / 2\right)^{-1} P_{2}(B)\left(H_{\infty}(B)-z-\omega / 2\right)^{-1}\right]_{s, s}(\mathbf{x}, \mathbf{x}) d \mathbf{x} .
\end{aligned}
$$

where $\Gamma$ is any finite contour such that $\Gamma \subset \rho\left(H_{\infty}(B)+\omega\right)$ for all $|\omega|<\frac{\left|d_{2}-d_{1}\right|}{4}$ and only enclosing the spectrum of $H_{\infty}(B)$ below $\frac{d_{1}+d_{2}}{2}$. Now the integrand in (4.12) is analytic in $\omega$ in a neighborhood of the origin. By expanding the resolvents one obtains:

$$
\begin{aligned}
& \sigma_{12}(B, T=0, \omega)= \\
& -\frac{e^{2}}{2 \pi m^{2}} \frac{1}{|\Omega|} \sum_{s=1}^{2} \int_{\Omega}\left\{\int_{\Gamma} \frac{1}{\omega} P_{1}(B)\left(H_{\infty}(B)-z\right)^{-1} P_{2}(B)\left(H_{\infty}(B)-z\right)^{-1}\right. \\
& +\frac{1}{2}\left[P_{1}(B)\left(H_{\infty}(B)-z\right)^{-1} P_{2}(B)\left(H_{\infty}(B)-z\right)^{-2}\right. \\
& \left.\left.-P_{1}(B)\left(H_{\infty}(B)-z\right)^{-2} P_{2}(B)\left(H_{\infty}(B)-z\right)^{-1}\right]\right\}_{s, s}(\mathbf{x}, \mathbf{x}) d \mathbf{x}+\mathcal{O}(\omega) .
\end{aligned}
$$


Apparently we have a first oder pole at $\omega=0$. But we now prove that the singular term in the r.h.s. of 4.13) is identically zero. Namely (when no spin variables appear the integral kernels below have to be understood as matrices in the spin space):

$$
\left\{\int_{\Gamma} P_{1}(B)\left(H_{\infty}(B)-z\right)^{-1} P_{2}(B)\left(H_{\infty}(B)-z\right)^{-1} d z\right\}(\mathbf{x}, \mathbf{x})=0 .
$$

Using the magnetic perturbation theory and the trick from (4.5) one can prove that even though the integrand in (4.14) has a quite singular kernel, after integration with respect to $z$ one gets a smooth kernel, exponentially localized near the diagonal (details will be given elsewhere).

Let us notice an operator equality which makes sense on compactly supported functions:

$$
\frac{1}{m}\left(H_{\infty}(B)-z\right)^{-1} P_{2}(B)\left(H_{\infty}(B)-z\right)^{-1}=i\left[X_{2},\left(H_{\infty}(B)-z\right)^{-1}\right],
$$

because the resolvent $\left(H_{\infty}(B)-z\right)^{-1}$ sends compactly supported functions into exponentially decaying functions (see (3.7)), which are in the domain of $X_{2}$. In fact, the operator on the right side has a nice integral kernel, given by

$$
\left\{i\left[X_{2},\left(H_{\infty}(B)-z\right)^{-1}\right]\right\}(\mathbf{x}, \mathbf{y})=i\left(x_{2}-y_{2}\right) G_{\infty}(\mathbf{x}, \mathbf{y} ; z),
$$

which is no longer singular at the diagonal and still exponentially localized near the diagonal, thus defining a bounded operator on the whole Hilbert space. After integration we get:

$$
\begin{aligned}
& \frac{i}{2 \pi} \int_{\Gamma} P_{1}(B)\left(H_{\infty}(B)-z\right)^{-1} P_{2}(B)\left(H_{\infty}(B)-z\right)^{-1} d z= \\
& \frac{i m}{2 \pi} \int_{\Gamma} P_{1}(B)\left[\left(H_{\infty}(B)-z\right)^{-1}, X_{2}\right] d z=m\left[P_{1}(B) \Pi^{B}, X_{2}\right],
\end{aligned}
$$

where we used that $P_{1}(B)$ and $X_{2}$ commute. Note that the magnetic perturbation theory states that the integral kernel of $P_{1}(B) \Pi^{B}$ is smooth and exponentially localized near the diagonal. Therefore $\left[P_{1}(B) \Pi^{B}, X_{2}\right]$ will have the integral kernel:

$$
\left\{\left[P_{1}(B) \Pi^{B}, X_{2}\right]\right\}(\mathbf{x}, \mathbf{y})=\left(y_{2}-x_{2}\right)\left\{P_{1}(B) \Pi^{B}\right\}(\mathbf{x}, \mathbf{y})
$$

which is identically zero at the diagonal and proves (4.14). We can conclude at this point that:

$$
\begin{aligned}
& \lim _{\omega \rightarrow 0} \sigma_{12}(B, T=0, \omega)= \\
& -\frac{e^{2}}{4 \pi m^{2}} \frac{1}{|\Omega|} \sum_{s=1}^{2} \int_{\Omega}\left\{P_{1}(B)\left(H_{\infty}(B)-z\right)^{-1} P_{2}(B)\left(H_{\infty}(B)-z\right)^{-2}\right. \\
& \left.-P_{1}(B)\left(H_{\infty}(B)-z\right)^{-2} P_{2}(B)\left(H_{\infty}(B)-z\right)^{-1}\right\}_{s, s}(\mathbf{x}, \mathbf{x}) d \mathbf{x} .
\end{aligned}
$$

Now consider the r.h.s. of (4.3). Since the magnetic field multiplying the spin will not change, our notation will only refer to $B_{2}$. Due to the stability 
of the spectrum against small variations of the magnetic field, for sufficiently small $\Delta B, \Pi^{B_{2}+\Delta B}$ still exists and

$$
\Pi^{B_{2}+\Delta B}-\Pi^{B_{2}}=\frac{i}{2 \pi} \int_{\Gamma}\left(\left(H_{\infty}\left(B_{2}+\Delta B\right)-z\right)^{-1}-\left(H_{\infty}\left(B_{2}\right)-z\right)^{-1}\right) d z .
$$

By using the magnetic perturbation theory [23] with respect to $\Delta B$ (see also the discussion around (6.2) ) one obtains:

$$
\begin{gathered}
{\left[\Pi^{B_{2}+\Delta B}-\Pi^{B_{2}}\right](\mathbf{x}, \mathbf{x})=\frac{i e \Delta B}{2 \pi m c} \int_{\Gamma}\left\{\int_{\mathbb{R}^{3}} d \mathbf{y}\left(H_{\infty}\left(B_{2}\right)-z\right)^{-1}(\mathbf{x}, \mathbf{y})\right.} \\
\left.\left[\mathbf{P}_{\mathbf{y}}\left(B_{2}\right) \cdot \mathbf{A}(\mathbf{y}-\mathbf{x})\right]\left(H_{\infty}\left(B_{2}\right)-z\right)^{-1}(\mathbf{y}, \mathbf{x})\right\} d z+\mathcal{O}\left((\Delta B)^{2}\right) .
\end{gathered}
$$

Now a very important identity is (see (3.3), 4.15) and (4.16)

$$
\begin{aligned}
& \mathbf{A}(\mathbf{y}-\mathbf{x})\left(H_{\infty}\left(B_{2}\right)-z\right)^{-1}(\mathbf{y}, \mathbf{x})= \\
& -\frac{i}{2 m} \mathbf{n}_{3} \wedge\left[\left(H_{\infty}\left(B_{2}\right)-z\right)^{-1} \mathbf{P}\left(B_{2}\right)\left(H_{\infty}\left(B_{2}\right)-z\right)^{-1}\right](\mathbf{y}, \mathbf{x}) .
\end{aligned}
$$

The remainder in $(\Delta B)^{2}$ will have a smooth integral kernel after the integration with respect to $z$, hence we obtain:

$$
\begin{aligned}
& \frac{\partial}{\partial B_{2}} \frac{1}{|\Omega|} \int_{\Omega} \Pi^{B}(\mathbf{x}, \mathbf{x}) d \mathbf{x}=-\frac{e}{4 \pi m^{2} c} \\
& \cdot \int_{\Omega}\left\{\int _ { \Gamma } \left[\left(H_{\infty}(B)-z\right)^{-1} P_{1}(B)\left(H_{\infty}(B)-z\right)^{-1} P_{2}(B)\left(H_{\infty}(B)-z\right)^{-1}\right.\right. \\
& \left.\left.\left.\quad-\left(H_{\infty}(B)-z\right)^{-1} P_{2}(B)\left(H_{\infty}(B)-z\right)^{-1} P_{1}(B)\left(H_{\infty}(B)-z\right)^{-1}\right) d z\right]\right\}(\mathbf{x}, \mathbf{x}) .
\end{aligned}
$$

From (4.23) and (4.19) we see that (4.3) follows if we can prove that one can circularly permute the operators under the integral sign in (4.23). One can prove this by interpreting (4.23) as the thermodynamic limit of the corresponding expression on finite volume and then using the invariance of the trace under cyclic permutations. Alternatively one can prove it directly and in what follows we outline the proof.

Due to the smoothing effect of the integral with respect to $z$, we can always restrict ourselves to considering a product of only two integral operators which commute with the discrete magnetic translations, and have kernels $e^{i b \phi_{\mathbf{a}}(\mathbf{x}, \mathbf{y})} K_{1}(\mathbf{x}, \mathbf{y})$ and $e^{i b \phi_{\mathbf{a}}\left(\mathbf{y}, \mathbf{x}^{\prime}\right)} K_{2}\left(\mathbf{y}, \mathbf{x}^{\prime}\right)$. We therefore look at an absolutely convergent integral of the form (the anti-symmetric magnetic phases disappear when we look at the diagonal, see (3.1)):

$$
\int_{\Omega} d \mathbf{x} \int_{\mathbb{R}^{3}} d \mathbf{y} K_{1}(\mathbf{x}, \mathbf{y}) K_{2}(\mathbf{y}, \mathbf{x})
$$

with

$$
K_{1,2}(\mathbf{x}, \mathbf{y})=K_{1,2}(\mathbf{x}+\vec{\gamma}, \mathbf{y}+\vec{\gamma})
$$


Then

$$
\begin{aligned}
& \int_{\Omega} d \mathbf{x} \int_{\mathbb{R}^{3}} d \mathbf{y} K_{1}(\mathbf{x}, \mathbf{y}) K_{2}(\mathbf{y}, \mathbf{x})=\sum_{\vec{\gamma} \in \mathcal{L}} \int_{\Omega} d \mathbf{x} \int_{\Omega} d \mathbf{y} K_{1}(\mathbf{x}, \mathbf{y}+\vec{\gamma}) K_{2}(\mathbf{y}+\vec{\gamma}, \mathbf{x}) \\
& =\sum_{\vec{\gamma} \in \mathcal{L}} \int_{\Omega} d \mathbf{x} \int_{\Omega} d \mathbf{y} K_{1}(\mathbf{x}-\vec{\gamma}, \mathbf{y}) K_{2}(\mathbf{y}, \mathbf{x}-\vec{\gamma}) \\
& =\int_{\Omega} d \mathbf{y} \int_{\mathbb{R}^{3}} d \mathbf{x} \int_{\mathbb{R}^{3}} K_{2}(\mathbf{y}, \mathbf{x}) K_{1}(\mathbf{x}, \mathbf{y})
\end{aligned}
$$

which gives the needed "trace cyclicity" and the theorem is proved.

We now turn to the question whether the limit in (4.3) actually vanishes as is suggested by some heuristic arguments (see e.g. 31]). We start by recalling some results about Wannier functions. Let $\sigma_{0}\left(B_{0}\right)$ be an isolated part of the spectrum of $H_{\infty}\left(B_{0}\right)$ and $\Pi_{0}^{B_{0}}$ the corresponding spectral projection. We say that $\Pi_{0}^{B_{0}}$ has a basis of exponentially localized (magnetic) Wannier functions if there exist $\alpha>0, w_{j} \in L^{2}\left(\mathbb{R}^{3}\right) \oplus L^{2}\left(\mathbb{R}^{3}\right), j=1,2, \ldots, p<\infty$ satisfying (we denote by $w_{j}(\mathbf{x}, s) \in \mathbb{C}$ the values of $w_{j}$ in $\mathbf{x} \in \mathbb{R}^{3}$ and $s \in\{1,2\}$ )

$$
\sum_{s=1}^{2} \int_{\mathbb{R}^{3}}\left|w_{j}(\mathbf{x}, s)\right|^{2} e^{2 \alpha|\mathbf{x}|} d \mathbf{x} \leq M<\infty,
$$

such that the set of functions $\left\{w_{j, \vec{\gamma}}\right\}_{j=1,2, \ldots p, \vec{\gamma} \in \mathcal{L}}$ with

$$
w_{j, \vec{\gamma}}(\mathbf{x}, s)=e^{i b \phi_{\mathbf{a}}(\mathbf{x}, \vec{\gamma})} w_{j}(\mathbf{x}-\vec{\gamma}, s)
$$

is a basis in the range of the projection $\Pi_{0}^{B_{0}}\left(L^{2}\left(\mathbb{R}^{3}\right) \oplus L^{2}\left(\mathbb{R}^{3}\right)\right)$. If the spin is neglected, it has been proved in 22 that the existence of bases of exponentially localized Wannier functions is stable against small values of the magnetic field (i.e. $B_{0}=0$ ). More precisely, if $\sigma_{0}$ is an isolated part of the spectrum of $-\Delta+V$ and the corresponding subspace has a basis of exponentially localized Wannier functions then, for sufficiently small $B, \sigma_{0}(B)$ is still isolated and the corresponding spectral subspace has a basis of exponentially localized magnetic Wannier functions. The methods in 21] together with the magnetic perturbation theory 13], 11], 23. allow one to generalize the above result to arbitrary $B_{0}$ and presence of the spin (as far as the spin-orbit term is sufficiently small) 12. Now the existence of exponentially localized magnetic Wannier functions for an isolated part of the spectrum and for the value of the magnetic field $B_{2}$ in an interval around $B_{0}$ allows one to write:

$$
\begin{aligned}
& \sum_{s=1}^{2} \int_{\Omega} \Pi_{0 ; s, s}^{B}(\mathbf{x}, \mathbf{x}) d \mathbf{x}=\int_{\Omega} \sum_{j, \vec{\gamma}} \sum_{s=1}^{2}\left|w_{j, \vec{\gamma}}(\mathbf{x}, s)\right|^{2} d \mathbf{x} \\
& =\sum_{j, \vec{\gamma}} \sum_{s=1}^{2} \int_{\Omega} d \mathbf{x}\left|w_{j}(\mathbf{x}-\vec{\gamma}, s)\right|^{2}=\sum_{j=1}^{p} \sum_{s=1}^{2} \int_{\mathbb{R}^{3}} d \mathbf{x}\left|w_{j}(\mathbf{x}, s)\right|^{2}=p .
\end{aligned}
$$

Thus the integrated density of states corresponding to the Fermi projection is constant in $B_{2}$ in a small interval around $B_{0}$, hence this band gives no contribution in the r.h.s. of (4.3).

For small fields, the above discussion can be summarized in: 
Theorem 4.2. Suppose $\left(d_{1}, d_{2}\right) \subset \rho(-\Delta+V), d_{2}>d_{1}$, and that the spectral subspace corresponding to $\left(-\infty, d_{1}\right]$ admits a basis of exponentially localized Wannier functions. Suppose that the spin-orbit interaction (see (2.2) is small enough such that as $c^{2}$ decreases from $\infty$ to its actual value, we have that $\frac{d_{1}+d_{2}}{2} \in \rho\left(H_{\infty}(0)\right)$. Then for sufficiently small $B$ :

$$
\lim _{\omega \rightarrow 0} \lim _{T \rightarrow 0} \sigma_{12}(B, T, \omega)=0 .
$$

In particular all the derivatives of $\sigma_{12}(B, 0)$ vanish for $B=0$, and this substantiates Roth's result (formula (50) in 31]) for the first order correction in $B$ at zero frequency.

\section{A closed formula for free electrons}

If $V=0$ it turns out that the conductivity tensor can be explicitly computed for all values of $B$ and $\omega$. The formula does not depend on whether we work in two or three dimensions. More precisely, we will show in this section that

$$
\sigma_{12}(B, \omega)=\frac{e^{3} n}{m^{2} c} \frac{B}{\omega^{2}-\frac{B^{2} e^{2}}{m^{2} c^{2}}},
$$

where $n=n(T, \mu, B)$ is the grand-canonical density. The formula (5.1) is well known in classical physics and goes back at least to Drude but we are not aware of a known fully quantum derivation. The coincidence of classical and quantum formulas can be understood taking into account that the Hamiltonians involved (choose the symmetric gauge) are quadratic and it is known that for this class of operators classical and quantum computations coincide in many instances. While it is possible to derive (5.1) by using the explicit form of the Green function or alternatively of eigenvalues and eigenprojections for the Landau Hamiltonian (see e.g. [18]) we shall obtain it below only using resolvent and commutation identities.

Let us only notice that when $\omega=0$ we re-obtain formula (18) in [34, while for a fixed frequency we get

$$
\frac{\partial \sigma_{12}}{\partial B}(0, \omega)=\frac{e^{3} n}{m^{2} c \omega^{2}}
$$

which is "the high frequency limit" or what Roth also calls "the free electron Faraday effect" in formula (51) from 31.

We begin by listing a few identities which are valid for a free electron on the 
entire space.

$$
\begin{aligned}
& i\left[P_{1}(B), P_{2}(B)\right]=\frac{B e}{c} \\
& i\left[H_{\infty}(B), P_{1}(B)\right]=-\frac{B e}{m c} P_{2}(B), \\
& i\left[H_{\infty}(B), P_{2}(B)\right]=\frac{B e}{m c} P_{1}(B), \\
& {\left[H_{\infty}(B),\left[H_{\infty}(B), P_{1}(B)\right]\right]=\frac{B^{2} e^{2}}{m^{2} c^{2}} P_{1}(B),} \\
& {\left[H_{\infty}(B),\left[H_{\infty}(B), P_{2}(B)\right]\right]=\frac{B^{2} e^{2}}{m^{2} c^{2}} P_{2}(B) .}
\end{aligned}
$$

Next, since in this case $\mathcal{A}_{s, s}^{\infty}(\mathbf{x}, \mathbf{x})$ does not depend upon $\mathbf{x}$ one has

$$
\begin{aligned}
& a(B, \omega)=-\frac{1}{2 \pi} \sum_{s=1}^{2} \\
& \left\{\int _ { \Gamma _ { \omega } } d z f _ { F D } ( z ) \left[P_{1}(B)\left(H_{\infty}(B)-z\right)^{-1} P_{2}(B)\left(H_{\infty}(B)-z-\omega\right)^{-1}\right.\right. \\
& +\quad z \rightarrow z-\omega]\}(\overrightarrow{0}, s ; \overrightarrow{0}, s) .
\end{aligned}
$$

Commuting $\left(H_{\infty}(B)-z-\omega\right)^{-1}$ with $P_{2}(B)$ in the first term, and $P_{1}(B)$ with $\left(H_{\infty}(B)-z+\omega\right)^{-1}$ in the second one, we obtain

$$
\begin{aligned}
& a(B, \omega)=-\frac{1}{2 \pi|\Omega|} \sum_{s=1}^{2} \\
& \left\{\int _ { \Gamma _ { \omega } } d z f _ { F D } ( z ) \left[P_{1}(B)\left(H_{\infty}(B)-z\right)^{-1}\left(H_{\infty}(B)-z-\omega\right)^{-1} P_{2}(B)\right.\right. \\
& +P_{1}(B)\left(H_{\infty}(B)-z\right)^{-1}\left(H_{\infty}(B)-z-\omega\right)^{-1} \\
& \cdot\left[H_{\infty}(B), P_{2}(B)\right]\left(H_{\infty}(B)-z-\omega\right)^{-1} \\
& +\left(H_{\infty}(B)-z+\omega\right)^{-1} P_{1}(B) P_{2}(B)\left(H_{\infty}(B)-z\right)^{-1} \\
& +\left(H_{\infty}(B)-z+\omega\right)^{-1}\left[H_{\infty}(B), P_{1}(B)\right] \\
& \left.\left.\quad \cdot\left(H_{\infty}(B)-z+\omega\right)^{-1} P_{2}(B)\left(H_{\infty}(B)-z\right)^{-1}\right]\right\}(\overrightarrow{0}, s ; \overrightarrow{0}, s) \\
& =I+I I+I I I+I V .
\end{aligned}
$$

Now $I+I I I$ can easily be computed. Indeed, by cyclic permutations (see 4.25) one can cluster the two resolvents and then by the resolvent identity

$$
\left(A-z_{1}\right)^{-1}\left(A-z_{2}\right)^{-1}=\left(z_{1}-z_{2}\right)^{-1}\left[\left(A-z_{1}\right)^{-1}-\left(A-z_{2}\right)^{-1}\right],
$$

one obtains four terms. Two of them vanish after the integration over $z$ due to 
the analyticity of the integrand while the other two give

$$
\begin{aligned}
& I+I I I=\frac{1}{2 \pi} \sum_{s=1}^{2} \\
& \left\{\int_{\Gamma_{\omega}} d z f_{F D}(z) \frac{1}{\omega}\left[P_{2}(B), P_{1}(B)\right]\left(H_{\infty}(B)-z\right)^{-1}\right\}(\overrightarrow{0}, s ; \overrightarrow{0}, s) \\
& =\frac{B e}{\omega} \sum_{s=1}^{2}\left\{f_{F D}\left(H_{\infty}(B)\right)\right\}(\overrightarrow{0}, s ; \overrightarrow{0}, s)=: \frac{B e}{\omega} n(T, \mu, B) .
\end{aligned}
$$

In an analogous manner

$$
\begin{aligned}
& I I I+I V=\frac{1}{2 \pi \omega} \sum_{s=1}^{2}\left\{\int_{\Gamma_{\omega}} d z f_{F D}(z)\right. \\
& \cdot\left\{\left(H_{\infty}(B)-z\right)^{-1}\left[H_{\infty}(B), P_{2}(B)\right]\left(H_{\infty}(B)-z-\omega\right)^{-1} P_{1}(B)\right. \\
& \left.-\left(H_{\infty}(B)-z\right)^{-1}\left[H_{\infty}(B), P_{1}(B)\right]\left(H_{\infty}(B)-z+\omega\right)^{-1} P_{2}(B)\right\} \\
& \}(\overrightarrow{0}, s ; \overrightarrow{0}, s) .
\end{aligned}
$$

At this point we commute $\left[H_{\infty}(B), P_{2}(B)\right]$ with $\left(H_{\infty}(B)-z-\omega\right)^{-1}$ in the first term, and $\left[H_{\infty}(B), P_{1}(B)\right]$ with $\left(H_{\infty}(B)-z+\omega\right)^{-1}$ in the second term and use (3.3) again. Some of the terms vanish after performing the integration over $z$ and the remaining ones write as:

$$
\begin{aligned}
& -\frac{1}{\omega}\left(H_{\infty}(B)-z\right)^{-1}\left[H_{\infty}(B), P_{2}(B)\right] P_{1}(B) \\
& -\frac{1}{\omega}\left(H_{\infty}(B)-z\right)^{-1}\left[H_{\infty}(B),\left[H_{\infty}(B), P_{2}(B)\right]\right]\left(H_{\infty}(B)-z-\omega\right)^{-1} P_{1}(B) \\
& -\frac{1}{\omega}\left(H_{\infty}(B)-z\right)^{-1}\left[H_{\infty}(B), P_{1}(B)\right] P_{2}(B) \\
& -\frac{1}{\omega}\left(H_{\infty}(B)-z\right)^{-1}\left[H_{\infty}(B),\left[H_{\infty}(B), P_{1}(B)\right]\right]\left(H_{\infty}(B)-z+\omega\right)^{-1} P_{2}(B) .
\end{aligned}
$$

Taking into account (5.2) the first and the third terms in (5.9) combine to

$$
-\frac{1}{\omega}\left(H_{\infty}(B)-z\right)^{-1}\left[H_{\infty}(B), P_{1}(B) P_{2}(B)\right]
$$

which after integration over $z$ is proportional to

$$
\begin{aligned}
& f_{F D}\left(H_{\infty}(B)\right) i\left[H_{\infty}(B), P_{1}(B) P_{2}(B)\right] \\
& =\frac{B e}{m c}\left\{f_{F D}\left(H_{\infty}(B)\right) P_{1}(B)^{2}-f_{F D}\left(H_{\infty}(B)\right) P_{2}(B)^{2}\right\},
\end{aligned}
$$

where we used the second and third identities in (5.2). Consider the unitary operator $U$ which implements the coordinate change $(U f)\left(x_{1}, x_{2}, x_{3}\right)=$ $f\left(x_{2},-x_{1}, x_{3}\right)$. Then one can prove that $U P_{1}(B) U^{*}=-P_{2}(B), U P_{2}(B) U^{*}=$ $P_{1}(B)$ and $U H_{\infty}(B) U^{*}=H_{\infty}(B)$. This implies that

$$
U f_{F D}\left(H_{\infty}(B)\right) P_{1}(B)^{2} U^{*}=f_{F D}\left(H_{\infty}(B)\right) P_{2}(B)^{2} .
$$


Since both operators have a smooth integral kernel, and because the rotation with $U$ does not change the diagonal value of the integral kernel on the left hand side, it means that the contribution given by (5.10) is zero.

Therefore we only remain with the second and fourth terms in (5.9). Using (5.2), they become:

$$
\begin{aligned}
& -\frac{B^{2} e^{2}}{m^{2} c^{2} \omega}\left(H_{\infty}(B)-z\right)^{-1} P_{2}(B)\left(H_{\infty}(B)-z-\omega\right)^{-1} P_{1}(B) \\
& -\frac{B^{2} e^{2}}{m^{2} c^{2} \omega}\left(H_{\infty}(B)-z\right)^{-1} P_{1}(B)\left(H_{\infty}(B)-z+\omega\right)^{-1} P_{2}(B) .
\end{aligned}
$$

Using once more the cyclicity of the trace and comparing with the starting point (5.3), we obtain the remarkable identity

$$
I I+I V=\frac{B^{2} e^{2}}{m^{2} c^{2} \omega^{2}} a(B, \omega) .
$$

Putting together (5.4), (5.6), and (5.12), we obtain the equation:

$$
a(B, \omega)=\frac{B e}{c \omega} n+\frac{B^{2} e^{2}}{m^{2} c^{2} \omega^{2}} a(B, \omega),
$$

which gives (5.1) (see (3.10).

\section{Magnetic perturbation theory and the linear term in $B$}

When $V \neq 0$ it is no longer possible to obtain a closed formula for $\sigma_{12}(B, \omega)$. Since in most physical applications the external magnetic field can be considered weak, an expansion in $B$ up to the first or second order would be sufficient. In this section we show that $a_{L}(B, \omega)$ has an expansion in $B$ to any order and write down the expressions of the first two terms. The first one gives the transverse conductivity at zero magnetic field and the second which is linear in $B$ provides the Verdet constant. From (3.6) and (3.8) (in what follows by $t r$ we mean the trace over the spin variable):

$$
\begin{aligned}
& a_{L}(B, \omega)=-\frac{1}{2 \pi\left|\Lambda_{L}\right|} \int_{\Lambda_{L}} d \mathbf{x}\left\{\operatorname{tr} \int_{\Gamma_{\omega}} d z f_{F D}(z)\right. \\
& \cdot \int_{\Lambda_{L}} d \mathbf{u}\left\{\left[\left(P_{\mathbf{x}, 1}(0)-b A_{1}(\mathbf{x}-\mathbf{u})\right) K_{L}(\mathbf{x}, \mathbf{u} ; z)\right]\right. \\
& \cdot\left[\left(P_{\mathbf{u}, 2}(0)-b A_{2}\left(\mathbf{u}-\mathbf{x}^{\prime}\right)\right) K_{L}\left(\mathbf{u}, \mathbf{x}^{\prime} ; z+\omega\right)\right] \\
& +\left[\left(P_{\mathbf{x}, 1}(0)-b A_{1}(\mathbf{x}-\mathbf{u})\right) K_{L}(\mathbf{x}, \mathbf{u} ; z-\omega)\right] \\
& \left.\left.\cdot\left[\left(P_{\mathbf{u}, 2}(0)-b A_{2}\left(\mathbf{u}-\mathbf{x}^{\prime}\right)\right) K_{L}\left(\mathbf{u}, \mathbf{x}^{\prime} ; z\right)\right]\right\}\right\}\left.\right|_{\mathbf{x}=\mathbf{x}^{\prime}}
\end{aligned}
$$

Let us mention here that one cannot interchange the order of the above integrals. First one performs the integral with respect to $\mathbf{u}$, then the integral in $z$, then we can put $\mathbf{x}=\mathbf{x}^{\prime}$ since the resulting kernel is smooth, and finally one integrates with respect to $\mathbf{x}$ over $\Lambda_{L}$.

When considering the expansion in $b$ of $a_{L}(B, \omega)$ we are left with the problem of the expansion of $K_{\Lambda_{L}}(\mathbf{x}, \mathbf{y} ; \zeta)$. This expansion is provided by the magnetic 
perturbation theory as developed in [23]. Following the steps in [23] in the case at hand one obtains:

$$
\begin{aligned}
& K_{L}(\mathbf{x}, \mathbf{y} ; z)=G_{L}^{(0)}(\mathbf{x}, \mathbf{y} ; z) \\
& +\frac{b}{m} \int_{\Lambda_{L}} G_{L}^{(0)}(\mathbf{x}, \mathbf{u} ; z)\left[\mathbf{P}_{\mathbf{u}}(0) \cdot \mathbf{A}(\mathbf{u}-\mathbf{y}) G_{L}^{(0)}(\mathbf{u}, \mathbf{y} ; z)\right] d \mathbf{u} \\
& +b \frac{g c \mu_{b}}{e} \int_{\Lambda_{L}} G_{L}^{(0)}(\mathbf{x}, \mathbf{u} ; z) \sigma_{3} G_{L}^{(0)}(\mathbf{u}, \mathbf{y} ; z) d \mathbf{u}+\mathcal{O}\left(b^{2}\right) \\
& =G_{L}^{(0)}(\mathbf{x}, \mathbf{y} ; z)+b G_{L}^{(o r b i t)}(\mathbf{x}, \mathbf{y} ; z)+b G_{L}^{(s p i n)}(\mathbf{x}, \mathbf{y} ; z)+\mathcal{O}\left(b^{2}\right) .
\end{aligned}
$$

The above integrands are matrices in the spin variable, that is why the spin does not appear explicitly. The error term $\mathcal{O}\left(b^{2}\right)$ can also be fully controlled with the magnetic perturbation theory (actually arbitrary order terms can be computed; see [22] for details). Plugging the expansion (6.2) into (6.2) and collecting the terms of zero and first order one obtains

$$
a_{L}(B, \omega)=a_{L}(0, \omega)+b a_{L, 1}(\omega)+\mathcal{O}\left(b^{2}\right),
$$

where the zeroth order term is:

$$
\begin{aligned}
& a_{L}(0, \omega)=-\frac{1}{2 \pi\left|\Lambda_{L}\right|} \int_{\Lambda_{L}} d \mathbf{x}\left\{\operatorname{tr} \int_{\Gamma_{\omega}} d z f_{F D}(z)\right. \\
& \left.\cdot\left\{P_{1}(0)\left(H_{L}(0)-z\right)^{-1} P_{2}(0)\left(H_{L}(0)-z+\omega\right)^{-1}+(z \rightarrow z-\omega)\right\}\right\}\left.\right|_{\mathbf{x}=\mathbf{x}^{\prime}},
\end{aligned}
$$

while the first order correction reads as:

$$
a_{L, 1}(\omega)=a_{L, 1}^{\text {orbit }}(\omega)+a_{L, 1}^{\text {spin }}(\omega),
$$

where

$$
\begin{aligned}
& a_{L, 1}^{\text {orbit }}(\omega)=-\frac{1}{2 \pi\left|\Lambda_{L}\right|} \int_{\Lambda_{L}} d \mathbf{x}\left\{\operatorname{tr} \int_{\Gamma_{\omega}} d z f_{F D}(z)\right. \\
& \cdot \int_{\Lambda_{L}} d \mathbf{u}\left\{-\left[A_{1}(\mathbf{x}-\mathbf{u}) G_{L}^{(0)}(\mathbf{x}, \mathbf{u} ; z)\right]\left[P_{\mathbf{u}, 2}(0) G_{L}^{(0)}\left(\mathbf{u}, \mathbf{x}^{\prime} ; z+\omega\right)\right]\right. \\
& -\left[P_{\mathbf{x}, 1}(0) G_{L}^{(0)}(\mathbf{x}, \mathbf{u} ; z)\right]\left[A_{2}\left(\mathbf{u}-\mathbf{x}^{\prime}\right) G_{L}^{(0)}\left(\mathbf{u}, \mathbf{x}^{\prime} ; z+\omega\right)\right] \\
& +\left[P_{\mathbf{x}, 1}(0) G_{L}^{(o r b i t)}(\mathbf{x}, \mathbf{u} ; z)\right]\left[P_{\mathbf{u}, 2}(0) G_{L}^{(0)}\left(\mathbf{u}, \mathbf{x}^{\prime} ; z+\omega\right)\right] \\
& +\left[P_{\mathbf{x}, 1}(0) G_{L}^{(0)}(\mathbf{x}, \mathbf{u} ; z)\right]\left[P_{\mathbf{u}, 2}(0) G_{L}^{(o r b i t)}\left(\mathbf{u}, \mathbf{x}^{\prime} ; z+\omega\right)\right] \\
& +(z \rightarrow z-\omega)\}\}\left.\right|_{\mathbf{x}=\mathbf{x}^{\prime}}, \\
& a_{L, 1}^{\text {spin }}(\omega)=-\frac{1}{2 \pi\left|\Lambda_{L}\right|} \int_{\Lambda_{L}} d \mathbf{x}\left\{\operatorname{tr} \int_{\Gamma_{\omega}} d z f_{F D}(z)\right. \\
& \cdot \int_{\Lambda_{L}} d \mathbf{u}\left\{\left[P_{\mathbf{x}, 1}(0) G_{L}^{(s p i n)}(\mathbf{x}, \mathbf{u} ; z)\right]\left[P_{\mathbf{u}, 2}(0) G_{L}^{(0)}\left(\mathbf{u}, \mathbf{x}^{\prime} ; z+\omega\right)\right]\right. \\
& +\left[P_{\mathbf{x}, 1}(0) G_{L}^{(0)}(\mathbf{x}, \mathbf{u} ; z)\right]\left[P_{\mathbf{u}, 2}(0) G_{L}^{(s p i n)}\left(\mathbf{u}, \mathbf{x}^{\prime} ; z+\omega\right)\right] \\
& +(z \rightarrow z-\omega)\}\}\left.\right|_{\mathbf{x}=\mathbf{x}^{\prime}} .
\end{aligned}
$$


Now consider the expression $\mathbf{A}(\mathbf{x}-\mathbf{y}) G_{L}^{(0)}(\mathbf{x}, \mathbf{y} ; z)$ appearing in the formula for $a_{L, 1}(\omega)$. Observing that it represents a commutator (see (3.3)) one has the identity

$$
\begin{aligned}
& \mathbf{A}(\mathbf{x}-\mathbf{y}) G_{L}^{(0)}(\mathbf{x}, \mathbf{y} ; z)=\left(\frac{1}{2} \mathbf{n}_{3} \wedge(\mathbf{x}-\mathbf{y})\right) G_{L}^{(0)}(\mathbf{x}, \mathbf{y} ; z) \\
& =\left(\frac{1}{2} \mathbf{n}_{3} \wedge\left[\mathbf{X},\left(H_{L}(0)-z\right)^{-1}\right]\right)(\mathbf{x}, \mathbf{y}) \\
& =-\frac{i}{2 m}\left\{\left(H_{L}(0)-z\right)^{-1}\left(\mathbf{n}_{3} \wedge P\right)\left(H_{L}(0)-z\right)^{-1}\right\}(\mathbf{x}, \mathbf{y}),
\end{aligned}
$$

where $\mathbf{X}$ denotes the multiplication operator with $\mathbf{x}$. By a straightforward (but somewhat tedious) computation one arrives at:

$$
a_{L, 1}(\omega)=a_{L, 1}^{\text {orbit }, 1}(\omega)+a_{L, 1}^{\text {orbit }, 2}(\omega)+a_{L, 1}^{\text {spin }}(\omega)
$$

where

$$
\begin{aligned}
a_{L, 1}^{\text {orbit }, 1}(\omega) & =\frac{i}{4 m \pi \omega\left|\Lambda_{L}\right|} \int_{\Lambda_{L}} d \mathbf{x}\left\{\operatorname{tr} \int_{\Gamma_{\omega}} d z f_{F D}(z)\right. \\
& \cdot\left[\sum_{\alpha=1}^{2} P_{\alpha}(0)\left(H_{L}(0)-z\right)^{-1} P_{\alpha}(0)\left(H_{L}(0)-z-\omega\right)^{-1}\right. \\
& +\sum_{\alpha=1}^{2} P_{\alpha}(0)\left(H_{L}(0)-z\right)^{-1} P_{\alpha}(0)\left(H_{L}(0)-z+\omega\right)^{-1} \\
& \left.\left.-\sum_{\alpha=1}^{2} P_{\alpha}(0)\left(H_{L}(0)-z\right)^{-1} P_{\alpha}(0)\left(H_{L}(0)-z\right)^{-1}\right]\right\}(\mathbf{x}, \mathbf{x}),
\end{aligned}
$$




$$
\begin{aligned}
a_{L, 1}^{\text {orbit }, 2}(\omega) & =\frac{i}{4 \pi m^{2}\left|\Lambda_{L}\right|} \int_{\Lambda_{L}} d \mathbf{x}\left\{\operatorname{tr} \int_{\Gamma_{\omega}} d z f_{F D}(z)\right. \\
& \cdot\left\{-P_{1}(0)\left(H_{L}(0)-z\right)^{-1} P_{1}(0)\left(H_{L}(0)-z\right)^{-1}\right. \\
& \cdot P_{2}(0)\left(H_{L}(0)-z\right)^{-1} P_{2}(0)\left(H_{L}(0)-z-\omega\right)^{-1} \\
& +P_{1}(0)\left(H_{L}(0)-z\right)^{-1} P_{2}(0)\left(H_{L}(0)-z\right)^{-1} \\
& \cdot P_{1}(0)\left(H_{L}(0)-z\right)^{-1} P_{2}(0)\left(H_{L}(0)-z-\omega\right)^{-1} \\
& -P_{1}(0)\left(H_{L}(0)-z+\omega\right)^{-1} P_{1}(0)\left(H_{L}(0)-z+\omega\right)^{-1} \\
& \cdot P_{2}(0)\left(H_{L}(0)-z+\omega\right)^{-1} P_{2}(0)\left(H_{L}(0)-z\right)^{-1} \\
& +P_{1}(0)\left(H_{L}(0)-z+\omega\right)^{-1} P_{2}(0)\left(H_{L}(0)-z+\omega\right)^{-1} \\
& \cdot P_{1}(0)\left(H_{L}(0)-z+\omega\right)^{-1} P_{2}(0)\left(H_{L}(0)-z\right)^{-1} \\
& -P_{1}(0)\left(H_{L}(0)-z\right)^{-1} P_{2}(0)\left(H_{L}(0)-z-\omega\right)^{-1} \\
& \cdot P_{1}(0)\left(H_{L}(0)-z-\omega\right)^{-1} P_{2}(0)\left(H_{L}(0)-z-\omega\right)^{-1} \\
& +P_{1}(0)\left(H_{L}(0)-z\right)^{-1} P_{2}(0)\left(H_{L}(0)-z-\omega\right)^{-1} \\
& \cdot P_{2}(0)\left(H_{L}(0)-z-\omega\right)^{-1} P_{1}(0)\left(H_{L}(0)-z-\omega\right)^{-1} \\
& -P_{1}(0)\left(H_{L}(0)-z+\omega\right)^{-1} P_{2}(0)\left(H_{L}(0)-z\right)^{-1} \\
& \cdot P_{1}(0)\left(H_{L}(0)-z\right)^{-1} P_{2}(0)\left(H_{L}(0)-z\right)^{-1} \\
& +P_{1}(0)\left(H_{L}(0)-z+\omega\right)^{-1} P_{2}(0)\left(H_{L}(0)-z\right)^{-1} \\
& \left.\left.\cdot P_{2}(0)\left(H_{L}(0)-z\right)^{-1} P_{1}(0)\left(H_{L}(0)-z\right)^{-1}\right\}\right\}(\mathbf{x}, \mathbf{x}),
\end{aligned}
$$

and

$$
\begin{aligned}
& a_{L, 1}^{\text {spin }}(\omega)=-\frac{g c \mu_{b}}{2 e \pi\left|\Lambda_{L}\right|} \int_{\Lambda_{L}} d \mathbf{x}\left\{\operatorname{tr} \int_{\Gamma_{\omega}} d z f_{F D}(z)\right. \\
& \cdot\left\{\left[P_{1}(0)\left(H_{L}(0)-z\right)^{-1} \sigma_{3}\left(H_{L}(0)-z\right)^{-1} P_{2}(0)\left(H_{L}(0)-z-\omega\right)^{-1}\right]\right. \\
& +\left[P_{1}(0)\left(H_{L}(0)-z\right)^{-1} P_{2}(0)\left(H_{L}(0)-z-\omega\right)^{-1} \sigma_{3}\left(H_{L}(0)-z-\omega\right)^{-1}\right] \\
& +\left[P_{1}(0)\left(H_{L}(0)-z+\omega\right)^{-1} \sigma_{3}\left(H_{L}(0)-z+\omega\right)^{-1} P_{2}(0)\left(H_{L}(0)-z\right)^{-1}\right] \\
& \left.\left.\quad+\left[P_{1}(0)\left(H_{L}(0)-z+\omega\right)^{-1} P_{2}(0)\left(H_{L}(0)-z\right)^{-1} \sigma_{3}\left(H_{L}(0)-z\right)^{-1}\right]\right\}\right\}(\mathbf{x}, \mathbf{x}) .
\end{aligned}
$$

\section{The periodic case}

Now consider the case when $V$ is periodic. In this case, after taking the thermodynamic limit one can replace (see (3.9) $) \frac{1}{\left|\Lambda_{L}\right|} \int_{\Lambda_{L}}$ with $\frac{1}{|\Omega|} \int_{\Omega}$ and rewrite 
(6.10) - 6.12) as integrals over the Brillouin zone

$$
\begin{aligned}
& a_{\infty, 1}^{\text {orbit,1 }}(\omega)=\frac{i}{4 m \pi \omega|\Omega|} \int_{\Omega *} d \mathbf{k} \int_{\Omega} d \mathbf{x}\left\{\operatorname{tr} \int_{\Gamma_{\omega}} d z f_{F D}(z)\right. \\
& \cdot \sum_{\alpha=1}^{2}\left(p_{\alpha}+k_{\alpha}\right)(h(\mathbf{k})-z)^{-1}\left(p_{\alpha}+k_{\alpha}\right)(h(\mathbf{k})-z-\omega)^{-1} \\
& +\sum_{\alpha=1}^{2}\left(p_{\alpha}+k_{\alpha}\right)(h(\mathbf{k})-z)^{-1}\left(p_{\alpha}+k_{\alpha}\right)(h(\mathbf{k})-z+\omega)^{-1} \\
& \left.-\sum_{\alpha=1}^{2}\left(p_{\alpha}+k_{\alpha}\right)(h(\mathbf{k})-z)^{-1} P_{\alpha}(0)(h(\mathbf{k})-z)^{-1}\right\}(\mathbf{x}, \mathbf{x}) \\
& a_{\infty, 1}^{\text {orbit, }}(\omega)=\frac{i}{4 \pi m^{2}|\Omega|} \int_{\Omega *} d \mathbf{k} \int_{\Omega} d \mathbf{x}\left\{\operatorname{tr} \int_{\Gamma_{\omega}} d z f_{F D}(z)\right. \\
& \cdot\left\{-\left(p_{1}+k_{1}\right)(h(\mathbf{k})-z)^{-1}\left(p_{1}+k_{1}\right)(h(\mathbf{k})-z)^{-1}\right. \\
& \cdot\left(p_{2}+k_{2}\right)(h(\mathbf{k})-z)^{-1}\left(p_{2}+k_{2}\right)(h(\mathbf{k})-z-\omega)^{-1} \\
& +\left(p_{1}+k_{1}\right)(h(\mathbf{k})-z)^{-1}\left(p_{2}+k_{2}\right)(h(\mathbf{k})-z)^{-1} \\
& \cdot\left(p_{1}+k_{1}\right)(h(\mathbf{k})-z)^{-1}\left(p_{2}+k_{2}\right)(h(\mathbf{k})-z-\omega)^{-1} \\
& -\left(p_{1}+k_{1}\right)(h(\mathbf{k})-z+\omega)^{-1}\left(p_{1}+k_{1}\right)(h(\mathbf{k})-z+\omega)^{-1} \\
& \cdot\left(p_{2}+k_{2}\right)(h(\mathbf{k})-z+\omega)^{-1}\left(p_{2}+k_{2}\right)(h(\mathbf{k})-z)^{-1} \\
& +\left(p_{1}+k_{1}\right)(h(\mathbf{k})-z+\omega)^{-1}\left(p_{2}+k_{2}\right)(h(\mathbf{k})-z+\omega)^{-1} \\
& \cdot\left(p_{1}+k_{1}\right)(h(\mathbf{k})-z+\omega)^{-1}\left(p_{2}+k_{2}\right)(h(\mathbf{k})-z)^{-1} \\
& -\left(p_{1}+k_{1}\right)(h(\mathbf{k})-z)^{-1}\left(p_{2}+k_{2}\right)(h(\mathbf{k})-z-\omega)^{-1} \\
& \cdot\left(p_{1}+k_{1}\right)(h(\mathbf{k})-z-\omega)^{-1}\left(p_{2}+k_{2}\right)(h(\mathbf{k})-z-\omega)^{-1} \\
& +\left(p_{1}+k_{1}\right)(h(\mathbf{k})-z)^{-1}\left(p_{2}+k_{2}\right)(h(\mathbf{k})-z-\omega)^{-1} \\
& \cdot\left(p_{2}+k_{2}\right)(h(\mathbf{k})-z-\omega)^{-1}\left(p_{1}+k_{1}\right)(h(\mathbf{k})-z-\omega)^{-1} \\
& -\left(p_{1}+k_{1}\right)(h(\mathbf{k})-z+\omega)^{-1}\left(p_{2}+k_{2}\right)(h(\mathbf{k})-z)^{-1} \\
& \cdot\left(p_{1}+k_{1}\right)(h(\mathbf{k})-z)^{-1}\left(p_{2}+k_{2}\right)(h(\mathbf{k})-z)^{-1} \\
& +\left(p_{1}+k_{1}\right)(h(\mathbf{k})-z+\omega)^{-1}\left(p_{2}+k_{2}\right)(h(\mathbf{k})-z)^{-1} \\
& \left.\left.\cdot\left(p_{2}+k_{2}\right)(h(\mathbf{k})-z)^{-1}\left(p_{1}+k_{1}\right)(h(\mathbf{k})-z)^{-1}\right\}\right\}(\mathbf{x}, \mathbf{x}) \text {, }
\end{aligned}
$$

and

$$
\begin{aligned}
& a_{\infty, 1}^{\operatorname{spin}}(\omega)=-\frac{g c \mu_{b}}{2 e \pi|\Omega|} \int_{\Omega *} d \mathbf{k} \int_{\Omega} d \mathbf{x}\left\{\operatorname{tr} \int_{\Gamma_{\omega}} d z f_{F D}(z)\right. \\
& \cdot\left\{\left[\left(p_{1}+k_{1}\right)(h(\mathbf{k})-z)^{-1} \sigma_{3}(h(\mathbf{k})-z)^{-1}\left(p_{2}+k_{2}\right)(h(\mathbf{k})-z-\omega)^{-1}\right]\right. \\
& +\left[\left(p_{1}+k_{1}\right)(h(\mathbf{k})-z)^{-1}\left(p_{2}+k_{2}\right)(h(\mathbf{k})-z-\omega)^{-1} \sigma_{3}(h(\mathbf{k})-z-\omega)^{-1}\right] \\
& +\left[\left(p_{1}+k_{1}\right)(h(\mathbf{k})-z+\omega)^{-1} \sigma_{3}(h(\mathbf{k})-z+\omega)^{-1}\left(p_{2}+k_{2}\right)(h(\mathbf{k})-z)^{-1}\right] \\
& \left.\left.+\left[\left(p_{1}+k_{1}\right)(h(\mathbf{k})-z+\omega)^{-1}\left(p_{2}+k_{2}\right)(h(\mathbf{k})-z)^{-1} \sigma_{3}(h(\mathbf{k})-z)^{-1}\right]\right\}\right\}(\mathbf{x}, \mathbf{x}) .
\end{aligned}
$$

Finally, for the convenience of the reader only interested in applying the theory to the case when one assumes that the Bloch bands and functions are 
known (as for example from Kohn-Luttinger type models), we write (7.1)-(7.3) in terms of Bloch functions and energies. The important thing here is that no derivatives with respect to the quasi-momentum appear. With the usual notation (here $\langle$,$\rangle denotes the scalar product over the spin variables):$

$$
\hat{\pi}_{i j}(\alpha, \mathbf{k})=\int_{\Omega}\left\langle u_{i}(\mathbf{x}, \mathbf{k}),\left(p_{\alpha}+\mathbf{k}_{\alpha}\right) u_{j}(\mathbf{x}, \mathbf{k})\right\rangle d \mathbf{x},
$$

and after some rearrangements, the terms coming from the orbital magnetism are:

$$
\begin{aligned}
& a_{\infty, 1}^{o r b i t, 1}(\omega)=\frac{1}{2 m \omega(2 \pi)^{3}} \sum_{\alpha=1}^{2} \int_{\Omega^{*}} d \mathbf{k}\left\{\sum_{j \geq 1}\left|\hat{\pi}_{j j}(\alpha, \mathbf{k})\right|^{2} f_{F D}^{\prime}\left(\lambda_{j}(\mathbf{k})\right)\right. \\
& \left.-\omega^{2} \sum_{j \neq l}\left|\hat{\pi}_{l j}(\alpha, \mathbf{k})\right|^{2} \frac{f_{F D}\left(\lambda_{j}(\mathbf{k})\right)-f_{F D}\left(\lambda_{l}(\mathbf{k})\right)}{\left[\left(\lambda_{j}(\mathbf{k})-\lambda_{l}(\mathbf{k})\right)^{2}-\omega^{2}\right]\left(\lambda_{j}(\mathbf{k})-\lambda_{l}(\mathbf{k})\right)}\right\}, \\
& a_{\infty, 1}^{\text {orbit }, 2}(\omega)=\frac{1}{2 m^{2}(2 \pi)^{3}} \int_{\Omega^{*}} d \mathbf{k} \sum_{n_{1}, n_{2}, n_{3}, n_{4} \geq 1} \frac{1}{2 \pi i} \int_{\Gamma_{\omega}} d z f_{F D}(z) \\
& \left\{\frac{\hat{\pi}_{n_{4} n_{1}}(1, \mathbf{k}) \hat{\pi}_{n_{1} n_{2}}(1, \mathbf{k}) \hat{\pi}_{n_{2} n_{3}}(2, \mathbf{k}) \hat{\pi}_{n_{3} n_{4}}(2, \mathbf{k})}{\left(z-\lambda_{n_{1}}(\mathbf{k})\right)\left(z-\lambda_{n_{2}}(\mathbf{k})\right)\left(z-\lambda_{n_{3}}(\mathbf{k})\right)\left(z+\omega-\lambda_{n_{4}}(\mathbf{k})\right)}\right. \\
& -\frac{\hat{\pi}_{n_{4} n_{1}}(1, \mathbf{k}) \hat{\pi}_{n_{1} n_{2}}(2, \mathbf{k}) \hat{\pi}_{n_{2} n_{3}}(1, \mathbf{k}) \hat{\pi}_{n_{3} n_{4}}(2, \mathbf{k})}{\left(z-\lambda_{n_{1}}(\mathbf{k})\right)\left(z-\lambda_{n_{2}}(\mathbf{k})\right)\left(z-\lambda_{n_{3}}(\mathbf{k})\right)\left(z+\omega-\lambda_{n_{4}}(\mathbf{k})\right)} \\
& +\frac{\hat{\pi}_{n_{4} n_{1}}(1, \mathbf{k}) \hat{\pi}_{n_{1} n_{2}}(1, \mathbf{k}) \hat{\pi}_{n_{2} n_{3}}(2, \mathbf{k}) \hat{\pi}_{n_{3} n_{4}}(2, \mathbf{k})}{\left(z-\omega-\lambda_{n_{1}}(\mathbf{k})\right)\left(z-\omega-\lambda_{n_{2}}(\mathbf{k})\right)\left(z-\omega-\lambda_{n_{3}}(\mathbf{k})\right)\left(z-\lambda_{n_{4}}(\mathbf{k})\right)} \\
& -\frac{\hat{\pi}_{n_{4} n_{1}}(1, \mathbf{k}) \hat{\pi}_{n_{1} n_{2}}(2, \mathbf{k}) \hat{\pi}_{n_{2} n_{3}}(1, \mathbf{k}) \hat{\pi}_{n_{3} n_{4}}(2, \mathbf{k})}{\left(z-\omega-\lambda_{n_{1}}(\mathbf{k})\right)\left(z-\omega-\lambda_{n_{2}}(\mathbf{k})\right)\left(z-\omega-\lambda_{n_{3}}(\mathbf{k})\right)\left(z-\lambda_{n_{4}}(\mathbf{k})\right)} \\
& +\frac{\hat{\pi}_{n_{4} n_{1}}(1, \mathbf{k}) \hat{\pi}_{n_{1} n_{2}}(2, \mathbf{k}) \hat{\pi}_{n_{2} n_{3}}(1, \mathbf{k}) \hat{\pi}_{n_{3} n_{4}}(2, \mathbf{k})}{\left(z-\lambda_{n_{1}}(\mathbf{k})\right)\left(z+\omega-\lambda_{n_{2}}(\mathbf{k})\right)\left(z+\omega-\lambda_{n_{3}}(\mathbf{k})\right)\left(z+\omega-\lambda_{n_{4}}(\mathbf{k})\right)} \\
& -\frac{\hat{\pi}_{n_{4} n_{1}}(1, \mathbf{k}) \hat{\pi}_{n_{1} n_{2}}(2, \mathbf{k}) \hat{\pi}_{n_{2} n_{3}}(2, \mathbf{k}) \hat{\pi}_{n_{3} n_{4}}(1, \mathbf{k})}{\left(z-\lambda_{n_{1}}(\mathbf{k})\right)\left(z+\omega-\lambda_{n_{2}}(\mathbf{k})\right)\left(z+\omega-\lambda_{n_{3}}(\mathbf{k})\right)\left(z+\omega-\lambda_{n_{4}}(\mathbf{k})\right)} \\
& +\frac{\hat{\pi}_{n_{4} n_{1}}(1, \mathbf{k}) \hat{\pi}_{n_{1} n_{2}}(2, \mathbf{k}) \hat{\pi}_{n_{2} n_{3}}(1, \mathbf{k}) \hat{\pi}_{n_{3} n_{4}}(2, \mathbf{k})}{\left(z-\omega-\lambda_{n_{1}}(\mathbf{k})\right)\left(z-\lambda_{n_{2}}(\mathbf{k})\right)\left(z-\lambda_{n_{3}}(\mathbf{k})\right)\left(z-\lambda_{n_{4}}(\mathbf{k})\right)} \\
& \left.-\frac{\hat{\pi}_{n_{4} n_{1}}(1, \mathbf{k}) \hat{\pi}_{n_{1} n_{2}}(2, \mathbf{k}) \hat{\pi}_{n_{2} n_{3}}(2, \mathbf{k}) \hat{\pi}_{n_{3} n_{4}}(1, \mathbf{k})}{\left(z-\omega-\lambda_{n_{1}}(\mathbf{k})\right)\left(z-\lambda_{n_{2}}(\mathbf{k})\right)\left(z-\lambda_{n_{3}}(\mathbf{k})\right)\left(z-\lambda_{n_{4}}(\mathbf{k})\right)}\right\} .
\end{aligned}
$$

As for the spin contribution, with the notation

$$
\hat{s}_{i j}(\mathbf{k}):=\int_{\Omega}\left\langle u_{i}(\mathbf{x}, \mathbf{k}), \sigma_{3} u_{j}(\mathbf{x}, \mathbf{k})\right\rangle d \mathbf{x},
$$


one has:

$$
\begin{aligned}
a_{\infty, 1}^{s p i n}(\omega) & =-\frac{g c \mu_{b}}{(2 \pi)^{4} e} \int_{\Omega^{*}} d \mathbf{k} \sum_{n_{1}, n_{2}, n_{3} \geq 1} \frac{1}{2 \pi i} \int_{\Gamma_{\omega}} d z f_{F D}(z) \\
& \left\{\frac{\hat{\pi}_{n_{1} n_{2}}(1, \mathbf{k}) \hat{s}_{n_{2} n_{3}}(\mathbf{k}) \hat{\pi}_{n_{3} n_{1}}(2, \mathbf{k})}{\left(\lambda_{n_{2}}(\mathbf{k})-z\right)\left(\lambda_{n_{3}}(\mathbf{k})-z\right)\left(\lambda_{n_{1}}(\mathbf{k})-z-\omega\right)}\right. \\
& +\frac{\hat{\pi}_{n_{1} n_{2}}(1, \mathbf{k}) \hat{\pi}_{n_{2} n_{3}}(2, \mathbf{k}) \hat{s}_{n_{3} n_{1}}(\mathbf{k})}{\left(\lambda_{n_{2}}(\mathbf{k})-z\right)\left(\lambda_{n_{3}}(\mathbf{k})-z-\omega\right)\left(\lambda_{n_{1}}(\mathbf{k})-z-\omega\right)} \\
& +\frac{\hat{\pi}_{n_{1} n_{2}}(1, \mathbf{k}) \hat{s}_{n_{2} n_{3}}(\mathbf{k}) \hat{\pi}_{n_{3} n_{1}}(2, \mathbf{k})}{\left(\lambda_{n_{2}}(\mathbf{k})-z+\omega\right)\left(\lambda_{n_{3}}(\mathbf{k})-z+\omega\right)\left(\lambda_{n_{1}}(\mathbf{k})-z\right)} \\
& \left.+\frac{\hat{\pi}_{n_{1} n_{2}}(1, \mathbf{k}) \hat{\pi}_{n_{2} n_{3}}(2, \mathbf{k}) \hat{s}_{n_{3} n_{1}}(\mathbf{k})}{\left(\lambda_{n_{2}}(\mathbf{k})-z+\omega\right)\left(\lambda_{n_{3}}(\mathbf{k})-z\right)\left(\lambda_{n_{1}}(\mathbf{k})-z\right)}\right\}
\end{aligned}
$$

\section{Conclusions}

We presented in the present paper a method which shed new light on the quantum dynamics/optical response in bulk media in the presence of a constant magnetic field. We applied the gauge invariant magnetic perturbation theory and gave a clear and very general way of dealing with long range magnetic perturbations.

The formal connection with the integer Quantum Hall effect was established in (4.2). Equations (6.9)-(6.12) and (7.4)-(7.8) contain our main result concerning the Verdet constant and the Faraday effect: it gives the linear term in $B$ of the transverse conductivity in terms of the zero magnetic field Green function. They open the way of using the recently developed Green function techniques for the calculation of optical and magneto-optical properties of solids, to the case when an external magnetic field is present. Our method can be applied to ordered, as well as to random systems (with the appropriate average over configurations). Of course, in the last case one has to assume ergodicity properties in order to insure convergence of results in the thermodynamic limit. Layers or other geometries can also be considered.

There are many subtle and difficult mathematical questions left aside in this paper, as those related to the thermodynamic limit, the convergence of infinite series over Bloch bands, and the low frequency limit when the Fermi energy lies in the spectrum. Another open problem is to consider self-interacting electrons and to investigate the exciton influence on the Faraday effect. These questions will be addressed elsewhere.

Our results are not only theoretical. In a future publication we will use the residue theorem in equations (7.4)-(17.8) to calculate the Verdet constant for various finite band models, and compare our results with the existing experimental data. Moreover, our results will be shown to imply those of Roth 31] and Nedoluha [20].

\section{Acknowledgments}

This paper has been written at the Department of Mathematical Sciences, Aalborg University. G.N. kindly thanks the Department for invitation and financial support. 
H.C. acknowledges support from the Statens Naturvidenskabelige Forskningsråd grant Topics in Rigorous Mathematical Physics, and partial support through the European Union's IHP network Analysis \& Quantum HPRN-CT2002-00277.

\section{References}

[1] Angelescu, N., Bundaru, M., and Nenciu, G.: "On the Landau diamagnetism", Commun. Math. Phys. 42, 9 (1975).

[2] Banhart, J.: "Optical Conductivity of Disordered Alloys Calculated from First Principles", Phys . Rev. Lett. 82, 2139 (1999).

[3] Bellissard, J., and Nakamura, S.: "Low energy bands do not contribute to quantum Hall effect", Commun. Math. Phys. 131, 283 (1990).

[4] Bennett, H.S., and Stern, E.A.: "Faraday effect in solids", Phys. Rev. 137, A448 (1965).

[5] Boswarva, I.M., Howard, R.E., and Lidiard, A.B.: "Faraday effect in semiconductors", Proc. Roy. Soc. (London) Series A. Mathematical and Physical Sciences, 269, 125 (1962).

[6] Boswarva, I.M., and Lidiard, A.B.: "Faraday effect in semiconductors.II", Proc. Roy. Soc. (London) Series A. Mathematical and Physical Sciences 278, 588-609 (1964)

[7] Bratelli, O., and Robinson, D.W. Operator Algebras and Quantum Statistical Mechanics 2 (Springer-Verlag, Berlin, Heidelberg, New York, Second Edition 1997)

[8] Bouclet, J-M, Germinet, F., Klein, A., and Schenker, J.H.: "Linear response theory for magnetic Schrdinger operators in disordered media", J. Funct. Anal. 226 no.2, 301 (2005).

[9] Butler, W.H.: "Theory of electronic transport in random alloys: KorringaKohn-Rostoker coherent-potential approximation", Phys . Rev. B 31, 3260 (1985).

[10] Combes J.M., and Thomas, L.: "Asymptotic behaviour of eigenfunctions for multiparticle Schrödinger operators", Commun. Math. Phys. 34, 251 (1973).

[11] Cornean, H.D.: "On the magnetization of a charged Bose gas in the canonical ensemble", Commun. Math. Phys. 202, 1 (2000).

[12] Cornean, H.D., Nenciu, A., and Nenciu, G., in preparation.

[13] Cornean, H.D., and Nenciu, G.: "On eigenfunction decay for twodimensional magnetic Schrödinger operators", Commun. Math. Phys. 198, 671 (1998).

[14] Cornean, H.D., and Nenciu, G., in preparation. 
[15] A. Gonis, Green Functions for Ordered and Disordered Systems (NorthHolland, Amsterdam, 1992).

[16] Huhne, T., and Ebert, H.: "Fully relativistic description of the magnetooptical properties of arbitrary layered systems", Phys . Rev. B 60,12982 (1999).

[17] Jimenez-Gonzales, H.J., Aggarwal, R.L., and Favrot, G.: "Infrared Faraday rotation of n-type InSb", Phys. Rev. B 49, 4571 (1994).

[18] Joynt, R., and Prange, R.: "Conditions for the quantum Hall effect", Phys. Rev. B84, 3303 (1984).

[19] Luttinger, J.M.: "The Effect of a Magnetic Field on Electrons in a Periodic Potential", Phys. Rev. 84, 814 (1951).

[20] Nedoluha, A.: "Perturbation-Theoretical Approach to Magneto-Optical Phenomena in the Spectral Region of Zero Absorption", Phys. Rev. 139, A1489 (1965).

[21] Nenciu, A., and Nenciu, G. : "Existence of exponentially localised Wannier functions for nonperiodic systems", Phys. Rev. B 47, 10112-10115 (1993).

[22] Nenciu, G.: "Dynamics of band electrons in electric and magnetic fields: rigorous justification of the effective Hamiltonians", Rev. Mod. Phys. 63, 91 (1991).

[23] Nenciu, G.: "On asymptotic perturbation theory for quantum mechanics: Almost invariant subspaces and gauge invariant magnetic perturbation theory", J. Math. Phys. 43, 1273 (2002).

[24] Nöckel, J.U., Stone, A.D., and Baranger, H.U.: "Adiabatic turn-on and the asymptotic limit in linear-response theory for open systems", Phys. Rev. B 48, 17569-17572 (1993).

[25] Pedersen, T.G.: "Tight-binding theory of Faraday rotation in graphite", Phys. Rev. B 68, 245104 (2003).

[26] Peierls, R.E.: "Zur Theorie des Diamagnetismus von Leitungelectronen", Z. Physik 80, 763 (1933).

[27] Perlov, A., Chadov, S., and Ebert, H.: "Green function approach for the ab initio calculation of the optical and magneto-optical properties of solids: Accounting for dynamical many-body effects", Phys . Rev. B 68, 245112 (2003).

[28] Pidgeon, C.R., and Brown, R.N.: "Interband Magneto-Absorption and Faraday Rotation in InSb", Phys . Rev. 146, 575 (1966).

[29] Roth A.P., and Fortin, E.: "Magneto-optics in semiconductor crystals of arbitrary orientation", Phys. Rev. B 18, 4200 (1978).

[30] Roth, L.M.: "Theory of Bloch electrons in a magnetic field", J.Phys. Chem. Solids 23, 433 (1962). 
[31] Roth, L.M.: "Theory of Faraday effect in solids", Phys. Rev. 133, A542 (1964).

[32] Schwinger, J.: "On Gauge Invariance and Vacuum Polarization", Phys. Rev. 82, 664 (1951).

[33] Sondheimer, E.H., and Wilson, A.H.: "The theory of the magnetoresistance effects in metals", Proc. Roy. Soc. (London) A 190, 435 (1947).

[34] Streda, P.: "Theory of quantized Hall conductivity in 2 dimensions", J. Phys. C.-Solid State Phys. 15, L717 (1982).

[35] Thouless, D.J.: "Wannier functions for magnetic sub-bands", J. Phys. C.Solid State Phys. 17, L325 (1984). 